\title{
Modelling of plasma rotation accounting for neutral beam injection and perturbation coils in JET and TEXTOR
}

\author{
A. Nicolai ${ }^{1}$, U. Daybelge ${ }^{2}$ and C. Yarim ${ }^{2}$ \\ ${ }^{1}$ Institut für Plasmaphysik, Forschungszentrum Jülich GmbH, Euratom Association, \\ Trilateral Euregio Cluster, D-52425 Jülich, Germany \\ ${ }^{2}$ Faculty of Aeronautics and Astronautics, Istanbul Technical University, 80626 Maslak, \\ Istanbul, Turkey
}

Received 14 November 2003, accepted for publication 7 May 2004

Published 28 May 2004

Online at stacks.iop.org/NF/44/S93

doi:10.1088/0029-5515/44/6/S10

\begin{abstract}
The impact of the helical perturbations, which can act as a momentum source or sink, on the rotation velocity is calculated on the basis of the ambipolarity constraint and the parallel momentum equation of the revisited neoclassical theory; this theory allows prediction of the parallel and poloidal flow speeds, $v_{\|}$, and $v_{\Theta}$, respectively, and therefore the radial electric field $E_{r}$ via the usual radial momentum balance equation.

Source terms account for the momentum deposition by neutral beam injection, pressure anisotropization and the $\vec{j} \times \vec{B}$ force density, the latter two due to Fourier components of (rotating) helical fields.

However, the neoclassical theory cannot account for the effect of the electrostatic turbulence on rotation in, e.g. TEXTOR L-modes. This is included by replacing the neoclassical viscosity $\eta_{2}$ by an anomalous one due to turbulence. The main results can be summarized as follows.

Using in the case of JET the data of shot \#59316 the maximum rotation speed can be reproduced with an accuracy of $10 \%$. A similar result is obtained for the TEXTOR shot \#91269.

If the angular velocity of the ( $m=2, n=1)$ Fourier component of the helical field is at low slip frequencies $\omega_{\mathrm{p}}-\omega_{\mathrm{f}}\left(\omega_{\mathrm{p}}\right.$ is the plasma rotation frequency and $\omega_{\mathrm{f}}$ the rotation frequency of the helical field $)$ gradually reduced to zero, a localized minimum appears and the gradient of the toroidal velocity becomes around $4 \times 10^{5} \mathrm{~s}^{-1}(\mathrm{JET})$.

However, if the slip frequency is larger than a critical value, the rotation profile of the rotation velocity is not influenced as observed at JET.

Although it is possible to create a large velocity shear around the singular surface, this shear is nonetheless limited by the reduction of the central velocity. Therefore, it might not be possible to trigger an ITB by plasma braking at the singular surface.
\end{abstract}

PACS numbers: 52.55.Fa, 52.30.-q

\section{Introduction}

Anomalous plasma transport and the concomitant deterioration of the confinement times of tokamaks much below the neoclassical prediction, in particular if auxiliary heating is applied, are key issues in fusion research.

Therefore, the surprising experimental discovery of the transition to a high confinement mode above a certain operating power has evoked considerable interest in improved confinement regimes, which seem to be related to toroidal and/or poloidal plasma spin-up and the rise of (sheared) radial electric fields [1-8].
The revisited neoclassical theory [9-13], which is based on Braginskii's equations [14,15], allows within the framework of a rigorous analytical approach calculation of the two dimensional velocity field on the flux surfaces and the perpendicular ambipolar electric field, i.e. the quantities which are believed to be important for the $\mathrm{L}-\mathrm{H}$ transition.

The theory is valid in collision dominated plasmas with steep gradients and was able to reproduce the toroidal spin-up in ALCATOR C-MOD [11, 12,26].

The importance of the theory is underlined by the fact that an interaction between the poloidal or toroidal spin-up and the turbulence-driven anomalous transport is believed to be a 
likely reason for the aforementioned $\mathrm{L}-\mathrm{H}$ mode transition in tokamaks.

It is also worthwhile to mention that ignition of the International Tokamak Experimental Reactor (ITER) requires an improved confinement regime.

In this contribution, a consistent modelling of the momentum transport is aimed at, which accounts for anomalous viscosity, neutral beam injection (NBI), friction due to recycled neutrals, braking due to pressure anisotropization and the momentum source due to $\vec{j} \times \vec{B}$ force density in the singular layer, the latter two evoked by the Fourier components of external nonaxisymmetric fields.

Particle and energy transport in general and stochastic transport due to these fields in particular are not considered; it is assumed instead these effects are included in the plasma background of the flat-top phase of the investigated experiments.

To account for the turbulence prevailing, e.g. in the L-mode of TEXTOR, the possibility is foreseen to introduce an anomalous viscosity [16]. Since the ion temperature gradient (ITG) threshold is exceeded in the TEXTOR L-mode, this instability, together with the trapped electron mode, are considered here as the main sources of turbulence.

The friction with the recycled neutral gas due to charge exchange is in limiter devices a strong source of momentum loss, as pointed out in [11].

The dominating friction of the rotating plasma with embedded islands generated by a radial magnetic field has already been discussed in [17]. There the radial magnetic field is assumed to be static. Here the case of a rotating radial field is envisaged as well.

In particular, at TEXTOR and JET static or rotating helical fields will be applied to influence the plasma rotation mainly driven by NBI. Here, the attempt is made to describe these experiments numerically.

This paper is organized as follows: in section 2 the main results of the revisited neoclassical theory are summarized and the characteristic quantities are given. Then the source terms due to NBI [16] (section 3), due to pressure anisotropization (section 4) and due to the $\vec{j} \times \vec{B}$ force density (section 5) are included in the neoclassical theory. Anomalous viscosity is introduced to achieve agreement with, e.g. TEXTOR results (section 6). An important basis of the analysis is the Fourier decomposition of the perturbing field (section 7). The standard solution method of the momentum equation and the necessary boundary conditions are summarized in section 8. Finally, results are presented concerning JET and TEXTOR in section 9 .

\section{Ambipolarity constraint and parallel momentum balance}

As mentioned earlier, the revisited neoclassical theory accounts, in particular, for steep temperature and density gradients at high collisionality. Since it is a collision dominated theory, it is based on the conservation equations for particles, momentum and energy [9-13]. For a two-component plasma with the velocities $\vec{u}_{j}$, the densities $n_{j}$, the particle sources
$S_{j}(\vec{x}, t)$, the friction forces $\vec{R}_{j}(x, t)$ and the momentum input $\vec{S}_{j}^{\mathrm{M}}(\vec{x}, t)(j=\mathrm{e}, \mathrm{i})$ we get

$$
\frac{\partial n_{j}}{\partial t}+\nabla \cdot\left(n_{j} \vec{u}_{j}\right)=S_{j}
$$

$m_{j} n_{j} \frac{\mathrm{d} \vec{u}_{j}}{\mathrm{~d} t}=-\nabla P_{j}-\nabla \cdot \overrightarrow{\vec{\Pi}}_{j}-e Z_{j} n_{j}\left(\vec{E}+\vec{u}_{j} \times \vec{B}\right)-\vec{R}_{j}+\vec{S}_{j}^{\mathrm{M}}$

where $P_{j}, \vec{E}$ and $\vec{B}$ are the scalar pressure, the electric and magnetic field, respectively. The viscous tensor is given by

$$
\overrightarrow{\vec{\Pi}}=-\eta_{0} \overrightarrow{\vec{W}}_{0}-\eta_{1}\left(\overrightarrow{\vec{W}}_{1}+4 \overrightarrow{\vec{W}}_{2}\right)+\eta_{3}\left(\overrightarrow{\vec{W}}_{3}+2 \overrightarrow{\vec{W}}_{4}\right)
$$

The tensors $\overrightarrow{\vec{W}}_{0}, \overrightarrow{\vec{W}}_{1}, \overrightarrow{\vec{W}}_{2}, \overrightarrow{\vec{W}}_{3}, \overrightarrow{\vec{W}}_{4}$, accounting for the parallel, perpendicular and gyro stress, and the viscosities $\eta_{0-3}$ are given in [14] (recently extended and completed by Mikhailovsky and Tsypin [15]). With the analogous terms $S_{j}^{\mathrm{E}}$ (sources) and $R_{j}^{\mathrm{E}}$ (frictional heating) we obtain for the power balance:

$$
\frac{3}{2} n_{j} \frac{\mathrm{d} T_{j}}{\mathrm{~d} t}+P_{j} \nabla \cdot \vec{u}_{j}=-\nabla \cdot \vec{q}_{j}-\overrightarrow{\vec{\Pi}}_{j}: \nabla \vec{u}_{j}+R_{j}^{\mathrm{E}}+S_{j}^{\mathrm{E}}
$$

In the revisited neoclassical theory [9], a plausible ordering inside the separatrix is introduced by means of a small expansion parameter $\mu \approx 0.1$ :

$$
\frac{c_{j}}{q R v_{j}} \approx \frac{L_{T}}{r} \approx \frac{r}{q R} \approx \frac{B_{\theta}}{B_{\phi}} \approx \mu
$$

and

$$
\frac{e_{j} V_{\mathrm{L}}}{T_{j}} \approx \sqrt{\frac{m_{\mathrm{e}}}{m_{\mathrm{i}}}} \approx \frac{a_{j}}{L_{T}} \approx \mu^{2}
$$

where $L_{T}=(\partial \ln T / \partial r)^{-1}$ is the radial scalelength of the temperature, $B_{\theta}$ and $B_{\phi}$ are the poloidal and toroidal fields, $v_{j}$, $c_{j}$ and $a_{j}$ are the collision frequency, the thermal speed and the gyro radius of the particle species $j$, respectively; $V_{\mathrm{L}}$ is the loop voltage, $m_{\mathrm{e}}$ the electron and $m_{\mathrm{i}}$ the ion mass

From the above ordering we conclude that the quantity

$$
\Lambda=\frac{\nu_{\mathrm{i}}}{\Omega_{\mathrm{i}}} \frac{q^{2} R^{2}}{L_{T} r} \approx 1
$$

and the quantity

$$
\Lambda^{*}=\sqrt{\frac{m_{\mathrm{e}}}{m_{\mathrm{i}}}}\left(\frac{q R v_{j}}{c_{j}}\right)^{2} \approx 1
$$

are both of the order of unity. $\Omega_{j}=c_{j} / a_{j}$ is the cyclotron frequency and $\Lambda$ measures the ratio of the contribution of the gyro-viscosity to the contribution of the parallel viscosity in equation (2). $\Lambda^{*}$ is the ratio of the ion heat diffusion time scale to the ion-electron equilibration time scale.

This means that the modifications of the revisited neoclassical theory with respect to the standard theory become important in the case of steep gradients (increasing the gyro-viscosity) and/or high collisionality.

For the toroidal and parallel momentum balances we use the magnetic field aligned orthonormal unit vectors:

- $\vec{p}$ in the radial direction (perpendicular to the flux surface),

- $\vec{b}$ in the geodesic direction (i.e. parallel to the flux surface and perpendicular to the field lines $)^{1}$, and

- $\vec{n}$ in the direction parallel to the field lines.

$1 \vec{b}$ is in the direction of the binormal if the fieldlines are geodesics which is normally not the case. 
These unit vectors can be expressed in terms of the unit vectors in the radial, poloidal and toroidal directions, $\vec{e}_{\psi}, \vec{e}_{\chi}$ and $\vec{e}_{\phi}$, respectively. Since we assume that these vectors are orthogonal (which is appropriate for axisymmetric systems) the metric tensor is diagonal and the line element is given by

$$
\mathrm{d} l^{2}=\left(h_{\psi} \mathrm{d} \psi\right)^{2}+\left(h_{\chi} \mathrm{d} \chi\right)^{2}+\left(h_{\phi} \mathrm{d} \phi\right)^{2}
$$

The Jacobian has the simple form $J=h_{\psi} h_{\chi} h_{\phi}$.

If the radial coordinate is identified with the flux function, the determination of the $\psi=$ const surfaces requires the solution of the Grad-Shafranov equation.

With the help of the coefficients $U_{\psi, j}^{\left(k_{1}\right)}, U_{\beta, j}^{\left(k_{2}\right)}(\psi)$ and $U_{\|, j}^{\left(k_{3}\right)}$ $\left(k_{1} \geqslant 6, k_{2} \geqslant 2, k_{3} \geqslant 1\right)$ which are all of the order of the thermal speed, the velocity components of species $j$ can be estimated as [9]

$$
\begin{gathered}
\vec{p} \cdot \vec{U}_{j}=\mu^{6} U_{\psi, j}^{(6)}(\psi)+\cdots \\
\vec{b} \cdot \vec{U}_{j}=\mu^{2} U_{\beta, j}^{(2)}(\psi)+\mu^{3} U_{\beta, j}^{(3)}(\psi, \chi)+\cdots \\
\vec{n} \cdot \vec{U}_{j}=\mu U_{\|, j}^{(1)}(\psi)+\mu^{2} U_{\|, j}^{(2)}(\psi, \chi)+\cdots
\end{gathered}
$$

This is mainly due to the diamagnetic velocity giving rise to a poloidal movement and the poloidal gradients, which drive a weak radial movement. The loop voltage evokes the movement of the ions relative to electrons [9].

Assuming that the magnetic field, density, temperature, potential, etc, are independent of the poloidal angle in dominant order, these quantities can be expanded in perturbation series as well. For example, the density $n$ and the magnetic field $B$ can be written as,

$$
n(\psi, \chi)=n^{(0)}(\psi)\left[1+n^{(1)}(\psi, \chi)+\cdots\right]
$$

and

$$
B(\psi, \chi)=B^{(0)}(\psi)\left[1+b^{(1)}(\psi, \chi)+\cdots\right]
$$

respectively. This procedure restricts the theory to large aspect ratio plasmas where the toroidal field is dominant.

We project equation (2) in the toroidal direction, evaluate the various contributions originating from the inertia and the viscous terms, integrate over a flux surface and get a time-dependent equation for the poloidally averaged toroidal speed $u_{\phi}$ :

$$
\begin{aligned}
& \int J h_{\phi} h_{\psi} \mathrm{d} \chi I_{\psi}=-\frac{m_{\mathrm{i}}}{e_{\mathrm{i}}} \frac{\partial}{\partial \psi}\left\{-2 \frac{P_{\mathrm{i}}^{(0)}}{B_{0}^{3}}\left[h_{\phi} B_{\phi} \frac{T_{\mathrm{i}}^{(0)}}{e_{\mathrm{i}} B_{0}}\right.\right. \\
& \left.\times\left(3 e_{\mathrm{i}} \frac{1}{T_{\mathrm{i}}^{(0)}} \frac{\partial V}{\partial \psi}+\frac{\partial \ln T_{\mathrm{i}}^{(0)}}{\partial \psi}-\frac{\partial \ln n_{\mathrm{i}}^{(0)}}{\partial \psi}+3 u_{\|, \mathrm{i}}^{(1)}\right)\right] \\
& \times \int n^{(1)} \frac{\partial b^{(1)}}{\partial \chi} \frac{\mathrm{d} \chi}{2 \pi}+12.48 \frac{P_{\mathrm{i}}^{(0)}}{B_{0}^{3}} \frac{B_{\chi}}{B_{0}} \frac{T_{\mathrm{i}}^{(0)}}{m_{\mathrm{i}} v_{\mathrm{i}}^{(0)} h_{\chi}^{(0)}} \\
& \left.\quad \times \int \frac{\partial n^{(1)}}{\partial \chi} \frac{\partial b^{(1)}}{\partial \chi} \frac{\mathrm{d} \chi}{2 \pi}+\frac{12}{10} \frac{P_{\mathrm{i}}^{(0)}}{B_{0}^{2}} \frac{1}{\Omega_{\mathrm{i}}^{(0)} \tau_{\mathrm{i}}^{(0)}} \frac{h_{\chi}^{(0)}}{B_{\phi}^{(0)} h_{\psi}^{(0)}} \frac{\partial u_{\|, \mathrm{i}}}{\partial \psi}\right\} \\
& \quad+\int J h_{\phi} \mathrm{d} \chi m_{\mathrm{i}} \frac{\partial n_{\mathrm{i}} u_{\phi, i}}{\partial t}=0
\end{aligned}
$$

Here $I_{\psi}$ is the radial charge flux density, the flux surface integral of which is zero because of ambipolarity. The index $i$ refers to the ions, $\tau_{\mathrm{i}}=1 / v_{\mathrm{i}}$ is the ion collision time. $n^{(1)}$ is given by [9]

$$
n^{(1)}=\alpha_{1} \cos (\chi)+\alpha_{2} \sin (\chi)
$$

where $\alpha_{1}, \alpha_{2}$ have been determined analytically in [9]. For $b^{(1)}$ we assume the Fourier expansion

$$
b^{(1)}=\epsilon\left(M_{1} \cos (\chi)+M_{2} \cos (2 \chi)+\cdots\right)
$$

where $\epsilon=r / R$ is the inverse aspect ratio. Here the radial coordinate $r$ is the effective radius defined by

$$
r=\sqrt{\frac{V(\psi)}{2 \pi^{2} R_{0}}}
$$

where $V(\psi)$ is the volume of the flux surface $\psi$.

In the case of circular cross-section we get $M_{1}=1$ analytically [9]. For elongated cross-sections $M_{1}$ can be evaluated numerically (appendix C).

Since in the case of elongated cross-sections the poloidal field is on top and bottom of the plasma weaker than in the equatorial plane (due to the vicinity of an X-point) one could expect a slight reduction of

$$
M_{1}=\frac{1}{\pi \epsilon} \int_{0}^{2 \pi} b^{(1)} \cos (\chi) \mathrm{d} \chi
$$

In the case of TEXTOR we get $M_{1}=1$ and the case of JET (shot \#59316) $M_{1}=0.95$. Both results are obtained numerically. Similar results are obtained for other values of $\kappa$. Therefore, we assume $M_{1}=1$ in the following.

We note that using the minor half axis ( $\frac{1}{2}$ of the extension of a flux surface in the equatorial plane) as radial coordinate ( $r=r^{\prime}$ as defined in the next section) would decrease $\epsilon$ by the factor $\approx 1 / \sqrt{\kappa}$ and thus increase $M_{1}$ by $\approx \sqrt{\kappa}$.

The other Fourier components $M_{k}, k>1$, do not contribute in equation (7) and are therefore not considered in the following.

The integrals

$$
\int n(1) \frac{\partial b^{(1)}}{\partial \chi} \frac{\mathrm{d} \chi}{2 \pi}=\frac{M_{1} \epsilon \alpha_{1}}{2}
$$

and

$$
\int \frac{\partial n^{(1)}}{\partial \chi} \frac{\partial b^{(1)}}{\partial \chi} \frac{\mathrm{d} \chi}{2 \pi}=-\frac{M_{1} \epsilon \alpha_{2}}{2}
$$

depend via $M_{1}$, in principle, on the flux surface geometry.

With the effective poloidal field

$$
B_{\theta}=\frac{1}{R_{0}} \frac{\mathrm{d} \psi}{\mathrm{d} r}
$$

the radial derivative $\partial / \partial \psi$ can be written as

$$
\frac{\partial}{\partial \psi}=\frac{1}{R_{0} B_{\theta}} \frac{\partial}{\partial r}
$$

Therefore, we get from equation (7) $\left(T=T_{\mathrm{i}}^{(0)}, n=n_{\mathrm{i}}^{(0)}\right)$

$$
\begin{gathered}
\frac{1}{r} \frac{\partial}{\partial r}\left[r \eta_{2}\left(\frac{\partial u_{\phi}}{\partial r}-M_{1} \frac{0.107 q^{2}}{1+\left(Q^{2} / S^{2}\right)} \frac{\partial \ln T}{\partial r} \frac{B_{\phi}}{B_{\theta}} u_{\theta}\right)\right] \\
=m_{\mathrm{i}} n_{\mathrm{i}}\left(\frac{\partial}{\partial t}+v_{\mathrm{cx}}\right) u_{\phi}+T_{\mathrm{NBI}}+T_{\mathrm{ANI}}+T_{j \times B}
\end{gathered}
$$


$u_{\theta}$ is the averaged poloidal speed. The perpendicular viscosity coefficient [11] is

$$
\eta_{2}=\frac{(6 / 5) n k T}{\Omega_{\mathrm{i}}^{2} \tau_{\mathrm{i}}}
$$

The velocities $Q$ and $S$ are defined by

$$
Q=\left[4 u_{\theta}-5 v_{n}\left(1+\frac{1}{2 \eta}\right)\right] \frac{B_{\phi}}{B}
$$

and

$$
S=\frac{8 v_{n} \eta}{\Lambda} \frac{B_{\phi}}{B}
$$

with the velocity $v_{n}=(T / e B)(\partial \ln (n) / \partial r)$ and the wellknown ratio

$$
\eta=\frac{\partial \ln (T) / \partial r}{\partial \ln (n) / \partial r}
$$

The charge exchange reactions are characterized by the charge exchange frequency

$$
v_{\mathrm{cx}}=\langle\sigma v\rangle_{\mathrm{cx}} n_{0}
$$

$\langle\sigma v\rangle_{\mathrm{cx}}$ is the rate coefficient for charge exchange, and $n_{0}$ the neutral gas density. The source terms $T_{\mathrm{NBI}}, T_{\mathrm{ANI}}, T_{j \times B}$ account for NBI, pressure anisotropization due to helical perturbations and for the $\vec{j} \times \vec{B}$-force at the singular surfaces. They are discussed in the following sections. We define the dimensionless quantities

$$
\begin{aligned}
g & =\frac{u_{\phi}}{v_{T}} \\
h & =\frac{u_{\theta}}{v_{T}}
\end{aligned}
$$

and

$$
x=\frac{r-r_{\text {in }}}{L_{\psi}}
$$

Here we used the (constant, positive) velocity

$$
v_{T}=\frac{1}{e B_{\phi}} \frac{T_{\text {in }}}{L_{\psi}}
$$

$T_{\text {in }}$ is the temperature at the point $P_{\text {in }}$ with radius $r_{\text {in }}$ and the length $L_{\psi}=L_{T}\left(r_{\text {in }}\right)$ is $L_{T}$ at $P_{\text {in }}$. In the case of ALCATOR C-MOD [11], $P_{\text {in }}$ is the 'inflection' point $P_{\text {inf }}$, which is defined in figure 1 as the locus of vanishing curvature of the ion temperature profile. In the case of TEXTOR (without temperature pedestal), $P_{\text {in }}$ is assumed to coincide with the plasma edge (i.e. $r_{\text {in }}=r_{\mathrm{s}}, r_{\mathrm{s}}$ is the minor plasma radius). The length $L_{\psi}=L_{T}\left(r_{\text {in }}\right)$ is $L_{T}$ at $P_{\text {in }}$. We get the dimensionless equation

$$
\frac{1}{x} \frac{1}{\eta_{2}} \frac{\partial}{\partial x}\left[x \eta_{2} G\right]=\hat{T}_{\mathrm{cx}}+\hat{T}_{\mathrm{NBI}}+\hat{T}_{\mathrm{ANI}}+\hat{T}_{j \times B}
$$

The quantity $G$ is given by

$$
G=\frac{\partial g}{\partial x}-\frac{0.107 q^{2}}{1+\left(Q^{2} / S^{2}\right)} \frac{\partial \ln T}{\partial x} \frac{B_{\phi}}{B_{\theta}} h
$$

The second term in equation (16) acts as a source term which is roughly proportional to $(\partial \ln T / \partial x) h$, i.e. to the temperature

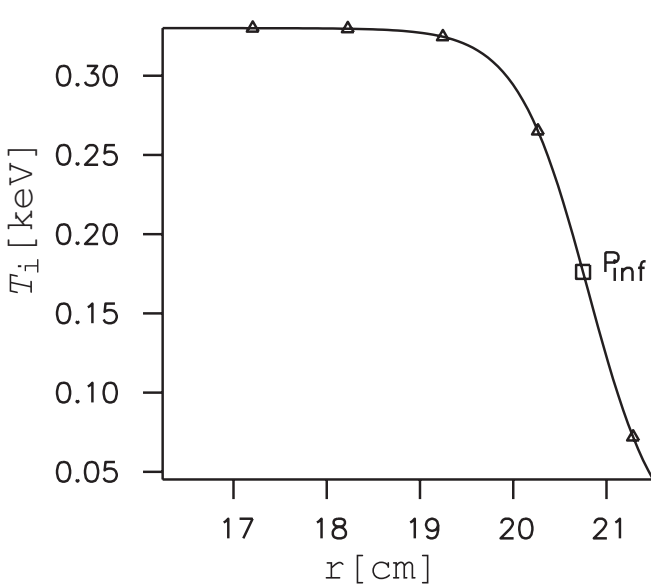

Figure 1. The steep temperature profile in the edge ALCATOR C-MOD has an inflection point $P_{\text {inf }}$ where the curvature vanishes. This point is taken as radial origin $(x=0)$ [12]. In the cases without temperature pedestals (TEXTOR and JET), $x=0$ is located at the plasma edge $r=r_{\mathrm{s}}$.

gradient. $q$ is the safety factor and $R$ the major radius. The dimensionless source term $\hat{T}_{\mathrm{cx}}$ is

$$
\hat{T}_{\mathrm{cx}}=t_{\mathrm{ci}} \frac{\hat{n}}{\hat{\eta}_{2}} v_{\mathrm{cx}} g
$$

It accounts for the aforementioned friction evoked by the neutral gas. The characteristic time $t_{\mathrm{c} \text {,in }}$ at the point $P_{\text {in }}$ is given by

$$
t_{\mathrm{c}, \text { in }}^{-1}=\frac{1}{m_{j} n_{\mathrm{i}} L_{\psi}^{2}} \eta_{2, \text { in }}
$$

and 'relative' viscosity $\hat{\eta}_{2}$ is defined by

$$
\eta_{2}=\hat{\eta}_{2} \eta_{2, \text { in }}
$$

where $\eta_{2, \text { in }}$ is the viscosity at the point $P_{\text {in }}$. Due to the scaling of $\tau_{\mathrm{i}}$ with density and temperature we get

$$
\hat{\eta}_{2}=\hat{n}^{2} \hat{T}^{-(1 / 2)+Z}
$$

$\hat{T}=T / T_{\text {in }}$ is the relative temperature and $\hat{n}=n / n_{\text {in }}$ the relative density. $T_{\text {in }}$ and $n_{\text {in }}$ are the temperature and density at the point $P_{\text {in }}$. As temperature dependence of $Z_{\text {eff }}$

$$
Z_{\mathrm{eff}}=Z_{\mathrm{eff}_{\mathrm{in}}} \hat{T}^{Z}
$$

is assumed, $Z$ is a constant.

Equation (15) is a second order equation for the normalized toroidal velocity $g$.

Using the rate coefficient for charge exchange with cold neutrals $(\approx 25 \mathrm{eV})\langle\sigma v\rangle_{\mathrm{cx}}=4.7 \times 10^{-14} \mathrm{~m}^{3} \mathrm{~s}^{-1}$, the charge exchange frequency of a neutral gas with the density $n_{0}\left(10^{19} \mathrm{~m}^{-3}\right)$ is given by

$$
v_{\mathrm{cx}}=4.7 \times 10^{-14}\left(\mathrm{~m}^{3} \mathrm{~s}^{-1}\right) \cdot n_{0}\left(10^{19} \mathrm{~m}^{-3}\right)=4.7 \times 10^{5} \cdot n_{0}
$$

We assume that the neutral gas density decays as $n_{0}=$ $N_{0} \exp \left[\left(L_{\psi} / L_{\text {neu }}\right) x\right] . N_{0}$ is the neutral density at the inflection point. $L_{\text {neu }} \approx 1 \mathrm{~cm}$ is an estimate of the decay length indicated by the SPUDNUT-code [28], assuming deuterium atoms only. 
Taking the parallel components of the terms in the momentum equation (2) and accounting in particular for the gyro-viscosity tensor [11] we get in the case of large aspect ratio and circular cross-section a nonlinear relation between the poloidal and toroidal plasma velocities [11].

$$
\begin{aligned}
h^{*}+ & 1.833=\frac{0.45 \Lambda}{1+\left(Q^{2} / S^{2}\right)}\left\{\frac{0.107 q^{2}}{1+\left(Q^{2} / S^{2}\right)} h^{*}+\frac{1}{2} \frac{1}{[\partial \hat{T} / \partial x]^{2}}\left(g^{*}\right)^{2}\right. \\
& -\frac{1}{\partial \hat{T} / \partial x} g^{*}\left[h^{*}-\left(1+\frac{2}{\eta}\right)\right] \\
& \left.+1.9\left[h^{*}-0.8\left(1+\frac{1.6}{\eta}\right)\right]^{2}\right\}
\end{aligned}
$$

Here, the definitions $g^{*}=\left(B_{\theta} / B_{\phi}\right)\left(u_{\phi} / \eta v_{n}\right)$, and $h^{*}=$ $u_{\theta} / \eta v_{n}$ are used, to cast equation (22) in a convenient form. In the case of elongated cross-sections, a more general form of equation (22) must be used in which similar integrals as in equation (7) are to be evaluated. These integrals contain the coefficient $M_{1}$ as a flux surface dependent part. We introduce the effective radius (8) and use $M_{1}=1$ as in equation (9).

Finally, we note that the magnetic field structure is prescribed and therefore island formation is neglected [11].

\section{Neutral beam injection}

Due to neutral injection [13] the particle number $N_{0}$ increases according to

$$
\dot{N}_{0}=\frac{I_{\mathrm{b}}[\mathrm{A}]}{e_{0}}=0.624 \times 10^{22} \frac{P_{\mathrm{b}}[\mathrm{MW}]}{E_{\mathrm{b}}[\mathrm{keV}]}
$$

where $I_{\mathrm{b}}$ is the injected current, $P_{\mathrm{b}}$ the injected power and $E_{\mathrm{b}}$ the beam energy. We concentrate on charge exchange reactions because they leave the particle number unchanged. The rate coefficient is given by

$$
\langle\sigma v\rangle_{\mathrm{cx}}=1.5 \times 10^{-13} \mathrm{~m}^{3} \mathrm{~s}^{-1}
$$

In a radial volume element $\Delta V=2 \pi R 2 \pi r \mathrm{~d} r$ during the time $\Delta t$ the momentum

$$
\Delta\left(m v_{\mathrm{i}} N_{\mathrm{i}}\right) \Delta V=m \dot{N}_{0} \Delta t \frac{\mathrm{d} l}{l_{\mathrm{mfp}}} v_{0}
$$

is deposited. $\mathrm{d} l$ is the line element along the beam path (figure 2), $v_{0}=\sqrt{E_{\mathrm{b}} / 2 m_{\mathrm{b}}}$ the speed of the neutrals and $l_{\mathrm{mfp}}$ the mean free path length due to charge exchange:

$$
l_{\mathrm{mfp}}=\frac{v_{0}}{\langle\sigma v\rangle_{\mathrm{cx}} N_{\mathrm{i}}}
$$

Let $\alpha$ be the angle between the beam tangency radius $R_{\mathrm{t}}$ and the straight line $R_{\mathrm{b}}$ connecting the centre point with the line element $\mathrm{d} l$. Then we have

$$
\cos \alpha=\frac{R_{\mathrm{t}}}{R_{\mathrm{b}}}
$$

and

$$
\frac{\mathrm{d} r^{\prime}}{\mathrm{d} l}=\sin \alpha \approx \sqrt{2 \frac{R_{\mathrm{b}}-R_{\mathrm{t}}}{R_{\mathrm{b}}}}=\sqrt{2 \epsilon^{\prime}}
$$

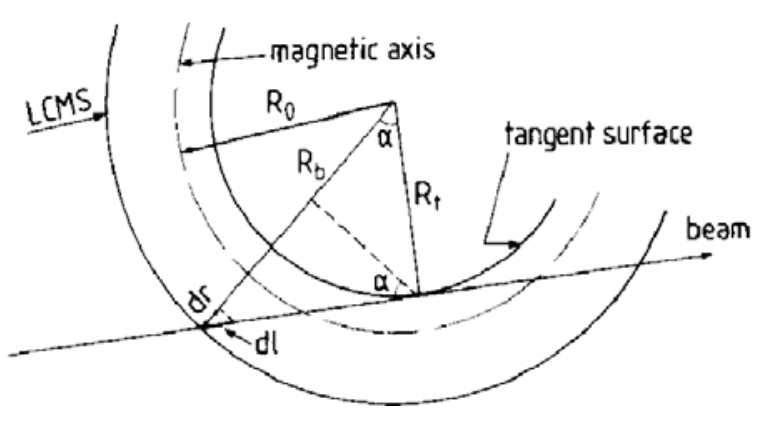

Figure 2. A pencil beam model is used to account for NBI. The beam 'tangency' radius is $R_{\mathrm{t}}$, the major radius of the line element $\mathrm{d} l$ along the beam is $R_{\mathrm{b}}$. $R_{\mathrm{t}}$ is assumed to be approximately equal to the radius of $R_{0}$ of the magnetic axis.

where $r^{\prime} \approx r / \sqrt{\kappa}$ is the minor half axis of the flux surface with effective radius $r$ and the elongation $\kappa$. $\epsilon^{\prime}$ is defined as $\epsilon^{\prime}=r^{\prime} / R_{0}$. Equation (24) entails

$$
\dot{v}_{\mathrm{i}} N_{\mathrm{i}}=\frac{\dot{N}_{0}\left(\mathrm{~d} l / l_{\mathrm{lmp}}\right) v_{0}}{2 \pi R 2 \pi r \mathrm{~d} r}
$$

Using the mean free path length (25) and assuming $R_{\mathrm{t}} \approx R_{0}$, we obtain

$$
\begin{gathered}
\dot{v}_{\mathrm{i}}\left[\mathrm{m} \mathrm{s}^{-2}\right]=\frac{\dot{N}_{0}\langle\sigma v\rangle_{\mathrm{cx}}}{2 \pi R_{0} 2 \pi r \sqrt{2 \epsilon^{\prime}} \sqrt{\kappa}} \\
=1.68 \times 10^{7} \frac{P_{\mathrm{MW}}}{E_{\mathrm{keV}}} \frac{1}{R_{0}[\mathrm{~m}] r[\mathrm{~m}] \sqrt{\epsilon}}
\end{gathered}
$$

The (dimensionless) source term is then

$$
\hat{T}_{\mathrm{INJ}}=t_{\mathrm{c}} \frac{\hat{n}}{\hat{\eta}_{2}} \frac{\dot{v}_{\mathrm{i}}}{v_{T}}
$$

We introduce $\hat{\eta}_{2}$ (equation (20)) and get

$$
\hat{T}_{\mathrm{INJ}}=t_{\mathrm{c}} \frac{1}{\hat{n} \hat{T}^{-(1 / 2)+Z}} \frac{\dot{v}_{\mathrm{i}}}{v_{T}}
$$

\section{Plasma braking due to pressure anisotropization at small slip frequencies $\omega$}

In this section, we deal with the case in which the plasma and the perturbing magnetic field, rotating with the frequency $\omega_{\mathrm{p}}$ and $\omega_{\mathrm{f}}$, respectively, have a small (absolute) slip frequency. This frequency is defined as

$$
\omega=\omega_{\mathrm{p}}-\omega_{\mathrm{f}}
$$

The plasma rotation frequency is

$$
\omega_{\mathrm{p}}=m \Omega_{\theta, \mathrm{p}}-n \Omega_{\phi, \mathrm{p}}
$$

and the helical field rotation frequency

$$
\omega_{\mathrm{f}}=m \Omega_{\theta, \mathrm{f}}-n \Omega_{\phi, \mathrm{f}}
$$

where $m, n$ are the poloidal and toroidal mode numbers, respectively. $\Omega_{\theta, \mathrm{p}}, \Omega_{\theta, \mathrm{f}}, \Omega_{\phi, \mathrm{p}}, \Omega_{\phi, \mathrm{f}}$ are the poloidal rotation frequency of the plasma, the poloidal rotation frequency of the helical field, the toroidal rotation frequency of the plasma and 
the toroidal rotation frequency of the helical field, respectively. The slip frequency is the frequency of the helical field seen in the rotating plasma system. If $\omega<0$, the helical field reduces the plasma rotation speed, for $\omega>0$ the opposite holds if the condition

$$
|\omega| \ll \omega_{\text {crit }}=\frac{-\Delta^{\prime}}{\tau_{\mathrm{si}}}
$$

is fulfilled. $\Delta^{\prime}$ and the time $\tau_{\mathrm{s}}$, both characteristics of the singular layer, are defined in section 5. Only for $|\omega| \ll \omega_{\text {crit }}$ helical perturbation imposed, e.g. by saddle coils at JET or the dynamic ergodic divertor (DED) at TEXTOR $[17,21,22]$ are assumed here to penetrate ideally because only feeble eddy currents are built up in the singular layer. Therefore it is assumed in the following that the perturbing field and the plasma are almost synchronized, i.e. that the slip frequency is small. Furthermore, without loss of generality, a stationary field is assumed $\left(\omega_{\mathrm{f}}=\Omega_{\theta, \mathrm{f}}=\Omega_{\phi, \mathrm{f}}=0\right)$.

Since the field of the perturbation coil has, in general, three components (linked by $\nabla \vec{B}=0$ ) the total field can be written as

$$
\begin{aligned}
\vec{B}= & B_{0, \phi} \vec{e}_{\phi}+B_{0, \theta} \vec{e}_{\theta}+\vec{e}_{\theta} \sum_{m, n} B_{\theta_{m, n}} \sin \left(m \theta-n \phi+\phi_{m, n}\right) \\
& +\vec{e}_{\phi} \sum_{m, n} B_{\phi_{m, n}} \sin \left(m \theta-n \phi+\phi_{m, n}\right) \\
& +\vec{e}_{\psi} \sum_{m, n} B_{\psi_{m, n}} \sin \left(m \theta-n \phi+\phi_{m, n}\right)
\end{aligned}
$$

where $B_{\theta_{m, n}}, B_{\phi_{m, n}}, B_{\psi_{m, n}}$ are the poloidal, toroidal and radial Fourier components of perturbation field.

The helical perturbations modulate the absolute value of the total field

$$
B=B_{0}(\theta)\left[1+\sum_{m, n} b_{m, n}(\theta, \phi)\right]
$$

where $B_{0}(\theta)=\sqrt{B_{0, \phi}^{2}+B_{0, \theta}^{2}}$ is the axisymmetric tokamak field and the $b_{m, n}(\theta, \phi)$ are given by

$$
b_{m, n}(\theta, \phi)=\frac{B_{\theta_{m, n}}+B_{\phi_{m, n}}}{\sqrt{B_{0, \phi}^{2}+B_{0, \theta}^{2}}} \sin \left(m \theta-n \phi+\phi_{m, n}\right)
$$

where $\phi_{m, n}$ are the phases. (The perturbing radial field generating the islands at the resonant surface in the case of ideal penetration, does not contribute to the modulation of the poloidal and toroidal field.)

Due to the pressure anisotropization we get as braking term $\left\langle\vec{e}_{\phi} \cdot \nabla \Pi\right\rangle=K v_{\phi}[24,27]$

$$
K=\frac{2 \sqrt{\pi} p_{\mathrm{i}}}{v_{T_{\mathrm{i}}}} \sum_{m, n}\left\langle\frac{\vec{e}_{\phi} \cdot \nabla B}{B} \frac{\partial b_{m, n}}{\partial \phi}\right\rangle \frac{q}{|m-n q|}
$$

This expression is derived for the plateau regime and it is valid in the vicinity of the rational surface with $q=m / n$. However, at the rational surface itself the Pfirsch-Schlüter regime is entered. In this regime the expression (37) must be modified, thus a singularity does not appear. More details are given in appendix A. We note that the penetration problem for $|\omega| \gg 0$ and also the torque due the helical field and the eddy currents will be addressed in section 5. The braking term due to helical perturbations (36) is given by [17]

$K v_{\phi}=\frac{\sqrt{\pi} p_{\mathrm{i}}}{v_{T_{\mathrm{i}}} R_{0}} \sum_{m, n} \frac{\left(B_{0, \theta} B_{\theta_{m, n}}+B_{0, \phi} B_{\phi_{m, n}}\right)^{2}}{B_{0}^{4}} \frac{n^{2} q}{|m-n q|} v_{\phi}$

In the case of helical conductors the dominant field components are the poloidal and the radial ones, because they are both perpendicular to the direction of the conductor. If we assume $b_{\theta_{m, n}} / B_{0} \approx 10^{-3}$ and the same parameters as the section before we get as braking time (since $B_{0, \theta} / B_{0} \approx \epsilon$ )

$$
\begin{aligned}
\tau & =\frac{R_{0}[\mathrm{~m}]}{\sqrt{\pi} 0.99 \times 10^{4} \sqrt{T_{\mathrm{i}}[\mathrm{eV}] / M}} 10^{6} \frac{|m-n q|}{n^{2} q \epsilon^{2}}[\mathrm{~s}] \\
& \approx 21 \frac{|m-n q|}{n^{2} q}[\mathrm{~s}]
\end{aligned}
$$

Because of singular denominator in the source term (proportional to $1 / \tau$ ) the braking time becomes small in the vicinity of the singular surface (at the singular surface the denominator must be modified according to appendix A). The large $\tau$ outside the rational surface means that the braking is only important at this surface.

In analogy to NBI, the dimensionless source term due to the braking by helical perturbations is given by

$$
T_{\mathrm{br}}=t_{\mathrm{ci}} \frac{\hat{n}}{\hat{\eta}_{2} \tau} g
$$

The radially dependent braking time, generalized to more Fourier components, is given by

$$
\begin{aligned}
\frac{1}{\tau}= & \frac{\sqrt{\pi} \times 10^{4} \sqrt{T_{\mathrm{i}}[\mathrm{eV}] / M}}{R_{0}[\mathrm{~m}]} \\
& \times\left[\sum_{m, n} \frac{n^{2} q}{|m-n q|} \frac{\left(B_{0, \theta} b_{\theta_{m, n}}+B_{0, \phi} b_{\phi_{m, n}}\right)^{2}}{B_{0}^{4}}\right]
\end{aligned}
$$

\section{Penetration of the helical field and plasma braking due to the $\vec{j} \times \vec{B}$ force density in the singular layer}

In general, the plasma rotates at a speed different from the perturbing field. Therefore the slip frequency $\omega$ (like in an induction motor) must be calculated. If the slip frequency is very large, no momentum transfer to the singular surface can be expected because of the eddy currents which prevent the penetration of the helical field. At low slip frequencies the eddy currents are small enough to allow the field to penetrate. The transferred momentum increases with increasing slip frequency until a maximum is reached. The dependence of the torque, transferred to the singular flux surface, on the slip frequency is given by [19]

$$
T_{\mathrm{EM}}=-\frac{8 \pi^{2} m^{3} R_{0}}{\mu_{0}} \frac{\omega \tau_{\mathrm{si}}}{\left(-\Delta^{\prime}\right)^{2}+\left(\omega \tau_{\mathrm{si}}\right)^{2}}\left|\Psi_{\mathrm{v}}\right|^{2}
$$

Here

$$
\tau_{\mathrm{si}}=\mu_{0} \sigma\left(r_{\mathrm{si}}\right) r_{\mathrm{si}} \delta_{\mathrm{si}}
$$

is the characteristic time of the singular layer with the conductivity $\sigma\left(r_{\mathrm{si}}\right)$

$$
\Delta^{\prime}=\left[r \frac{\mathrm{d} \psi_{\text {plasma }}}{\mathrm{d} r}\right]_{r_{\mathrm{s}-}}^{r_{\mathrm{s}+}}
$$


is called the 'tearing mode stability index' because $\Delta^{\prime}$ is proportional to the growth rate of the tearing mode islands. $\Delta^{\prime}$ can be inferred from the ideal tearing mode equation

$$
\frac{1}{r} \frac{\mathrm{d}}{\mathrm{d} r}\left(r \frac{\mathrm{d} \psi}{\mathrm{d} r}\right)-\frac{m^{2}}{r^{2}} \psi-\frac{\mu_{0} m(\mathrm{~d} j / \mathrm{d} r)}{B_{\theta}(m-n q)} \psi=0
$$

The solution procedure consists in setting up of the boundary conditions and the integration of equation (44) from the inside $(r=0)$ to the singular layer $\left(r=r_{\mathrm{si}}-\epsilon\right)$ and from the outside until the singular layer $\left(r=r_{\mathrm{si}}+\epsilon\right)$. $\epsilon$ is a small number. The boundary conditions are $\psi=0, \mathrm{~d} \psi / \mathrm{d} r=0$ at $r=0$ and $\psi \approx 0$ at $r=k r_{\mathrm{s}}$ ( $k$ is a number in the range $k>2) . \quad(\mathrm{d} \psi / \mathrm{d} r)\left(r_{\mathrm{s}}\right)$ is adjusted in such a way that $\psi\left(r_{\mathrm{si}}-\epsilon\right)=\psi\left(r_{\mathrm{si}}+\epsilon\right) . \quad j(r)$ is the plasma current density which is parabolic in $r$. For $k>2, \Delta^{\prime}$ becomes almost independent of $k$.

We assume in this section $\Delta^{\prime}<0$ so that the plasma is tearing mode stable.

$\Psi_{\mathrm{v}}$ in equation (42) is the vacuum flux function due external coils, at the singular layer:

$$
\Psi_{\mathrm{v}}=\psi\left(r_{\mathrm{si}}\right) \exp \left[\mathrm{i}\left(m \theta-n \phi+\omega_{\mathrm{f}} t\right)\right]
$$

where $\psi(r)$ is the radial part of the vacuum flux function. At the singular surface we have the flux function [19]

$$
\Psi_{\mathrm{si}}=\Psi_{\mathrm{v}} \frac{2 m}{-\Delta^{\prime}+\mathrm{i} \omega \tau_{\mathrm{si}}}
$$

$\Psi_{\text {si }}$ differs from $\Psi_{\mathrm{v}}$ because of the eddy currents. Real and imaginary parts of expression (46) allow, e.g. to compute the phase shift between $\Psi_{\mathrm{si}}$ and $\Psi_{\mathrm{v}}$. For static fields, the phase shift vanishes and $\Psi_{\mathrm{si}}=\Psi_{\mathrm{v}}\left(2 \mathrm{~m} /\left(-\Delta^{\prime}\right)\right)$ is in general larger than $\Psi_{\mathrm{v}}$ ('amplification'). At high slip frequencies, beyond the critical frequency

$$
\omega_{\text {crit }}=\frac{-\Delta^{\prime}}{\tau_{\mathrm{si}}}
$$

the flux function inside the plasma is considerably lower than $\Psi_{\mathrm{v}}$.

Since the torque is deposited in the volume $4 \pi^{2} r r_{\mathrm{si}} \delta_{\mathrm{si}} R_{0}$ with the radial extension $\delta_{\mathrm{si}}$, the momentum density transferred to the plasma is given by

$$
\begin{gathered}
T_{j \times B}^{*}=T_{\mathrm{EM}}^{*}=\frac{T_{\mathrm{EM}}}{4 \pi^{2} r r_{\mathrm{si}} \delta_{\mathrm{si}} R_{0}} \\
=-\frac{8 \pi^{2} m^{3} R_{0}}{\mu_{0} 4 \pi^{2} r r_{\mathrm{si}} \delta_{\mathrm{si}} R_{0}} \frac{\omega \tau_{\mathrm{si}}}{\left(-\Delta^{\prime}\right)^{2}+\left(\omega \tau_{\mathrm{si}}\right)^{2}}\left|\Psi_{\mathrm{v}}\right|^{2} \\
\text { in } r_{\mathrm{si}}-\delta_{\mathrm{si}} / 2<r<r_{\mathrm{si}}+\delta_{\mathrm{si}} / 2 \text { and } \\
T_{j \times B}^{*}=0
\end{gathered}
$$

elsewhere.

The width of the layer can be written as [19]

$$
\delta_{\mathrm{si}}=2.104 r_{\mathrm{si}}\left[\frac{\tau_{\mathrm{h}}^{2}}{\tau_{\mathrm{R}} \tau_{\mathrm{V}}}\right]^{1 / 6}
$$

Here, the hydrodynamic time is

$$
\tau_{\mathrm{h}}=\frac{R_{0}}{r_{\mathrm{si}} n s} \tau_{\mathrm{a}}
$$

where $\tau_{\mathrm{a}}$ is the Alfvén-time given by

$$
\tau_{\mathrm{a}}=\frac{r_{\mathrm{si}}}{v_{\mathrm{a}}}=\frac{r_{\mathrm{si}}}{\sqrt{B_{0}^{2} / n_{\mathrm{si}} m_{\mathrm{i}} \mu_{0}}}=r_{\mathrm{si}} \sqrt{\frac{\left(n_{\mathrm{si}} m_{\mathrm{i}} \mu_{0}\right)}{B_{0}^{2}}}
$$

where $n_{\mathrm{si}}$ is the ion density at the singular layer.

The shear parameter $s$ is defined by

$$
s=\frac{r q^{\prime}}{q}
$$

The viscous diffusion time can be expressed as

$$
\tau_{\mathrm{V}}=\frac{r_{\mathrm{si}}^{2} m_{\mathrm{i}} n_{\mathrm{si}}}{\eta_{2}\left(r_{\mathrm{si}}\right)}
$$

Finally, the resistive time is

$$
\tau_{\mathrm{R}}=\mu_{0} r_{\mathrm{si}}^{2} \sigma\left(r_{\mathrm{si}}\right)
$$

The braking time, i.e. the time needed to brake down a plasma moving with the speed $v_{\phi}$ is then given by

$$
\begin{aligned}
\tau= & \frac{n_{\mathrm{si}} m_{\mathrm{i}} v_{\phi}}{T_{j \times B}^{*}}=\left[n_{\mathrm{si}} m_{\mathrm{i}} v_{\phi}\right] \\
& \times\left[\frac{8 \pi^{2} m^{3} R_{0}}{r \mu_{0} 4 \pi^{2} r_{\mathrm{si}} R_{0} \delta_{\mathrm{si}}} \frac{\omega \tau_{\mathrm{si}}}{\left(-\Delta^{\prime}\right)^{2}+\left(\omega \tau_{\mathrm{si}}\right)^{2}}\left|\Psi_{\mathrm{v}}\right|^{2}\right]^{-1}
\end{aligned}
$$

This expression is modified as follows: since the radial component of the helical perturbation is $B_{\mathrm{hel}_{r}}=(\mathrm{m} / \mathrm{r}) \psi_{\mathrm{v}}$, we get

$$
\begin{aligned}
\tau= & n_{\mathrm{si}} m_{\mathrm{i}}\left[\left(\frac{v_{T}^{2}}{v_{T}}\right)\left(\frac{v_{\phi}}{v_{T}}\right) m^{2}\right] \\
& \times\left[\frac{16 \pi^{2} m^{3} R_{0}}{r 2 \mu_{0} 4 \pi^{2} r_{\mathrm{si}} R_{0} \delta_{\mathrm{si}}} \frac{\omega \tau_{\mathrm{si}}}{\left(-\Delta^{\prime}\right)^{2}+\left(\omega \tau_{\mathrm{si}}\right)^{2}}\left|r B_{\mathrm{hel}_{r}}\right|^{2}\right]^{-1}
\end{aligned}
$$

Analogously to the local poloidal $\beta_{\mathrm{pol}}=2 \mu_{0} P / B_{\theta}^{2}$ ( $P$ is the total local pressure) we define

$$
\beta_{\mathrm{fi}}=\frac{n_{\mathrm{si}} m_{\mathrm{i}} v_{T}^{2} 2 \mu_{0}}{B_{\text {hel }_{r}}^{2}}
$$

and obtain

$$
\tau=\frac{\left(\delta_{\mathrm{si}} / v_{T}\right)\left(v_{\phi} / v_{T}\right) \beta_{\mathrm{fi}}}{\left(4 m^{3} r / r_{\mathrm{si}}\right)\left(\omega \tau_{\mathrm{si}} /\left(\left(-\Delta^{\prime}\right)^{2}+\left(\omega \tau_{\mathrm{si}}\right)^{2}\right)\right)}
$$

The source term is given by

$$
T_{\mathrm{EM}}^{*}=\frac{v_{\phi} n_{\mathrm{si}} m_{\mathrm{i}}}{\tau}=\frac{n_{\mathrm{si}} m_{\mathrm{i}} 4 m^{3} r}{r_{\mathrm{si}} \beta_{\mathrm{fi}}} \frac{\omega \tau_{\mathrm{si}}}{\left(-\Delta^{\prime}\right)^{2}+\left(\omega \tau_{\mathrm{si}}\right)^{2}} \frac{v_{T}^{2}}{\delta_{\mathrm{si}}}
$$

To get the dimensionless source term $\hat{T}_{\mathrm{EM}}^{*}$ in analogy to equation (28), the source term must be multiplied with $t_{\text {ci }} / v_{T} n_{\text {inf }} m_{\mathrm{i}} \hat{\eta}_{2}$ :

$$
\hat{T}_{\mathrm{EM}}^{*}=\frac{\hat{n}_{\mathrm{si}} 4 m^{3} r}{r_{\mathrm{si}} \beta_{\mathrm{fi}} \hat{\eta}_{2}} \frac{\omega \tau_{\mathrm{si}}}{\left(-\Delta^{\prime}\right)^{2}+\left(\omega \tau_{\mathrm{si}}\right)^{2}} \frac{v_{T}}{\delta_{\mathrm{si}}} t_{\mathrm{ci}}
$$

for the numerical treatment the discontinuous source term defined by the equations (47) and (48) is replaced by the expression

$$
T_{j \times B}^{\mathrm{c}}=T_{\mathrm{EM}}^{*} C_{n} \exp \left[-\left(\frac{r-r_{\mathrm{si}}}{\delta_{\mathrm{si}}}\right)^{2}\right]
$$

where $C_{n}=1 / \sqrt{\pi} \delta_{\mathrm{si}}$ is obtained from the requirement

$$
\int T_{j \times B}^{*} \mathrm{~d} r=\int T_{j \times B}^{\mathrm{c}} \mathrm{d} r
$$




\section{Anomalous viscosity}

Attempts to reproduce the rotation velocity of the L-mode TEXTOR plasma with the classical viscosity fail because the obtained velocities are much too large. Therefore, like in the case of the heat conduction, anomalous viscosity must be envisaged [16]. The shape of the temperature and density profiles in TEXTOR make the ITG instability likely to be the primary candidate for anomalous viscosity. The growth rate of the instability is [16]

$$
\gamma_{\mathrm{ITG}}=\frac{2 c T_{\mathrm{e}} k_{y}}{e B R} \sqrt{\frac{\zeta}{\langle Z\rangle}(1-0.67 p)-\frac{\zeta^{2} p^{2}}{4}}
$$

Here, the peaking factor $p$ is defined by $p=1 / \eta$ and $\zeta=-(R / 2)(\mathrm{d} \ln T / \mathrm{d} r)$. The parameter $\eta$ is defined by equation (13). In the case of saturated turbulence the diffusivity is given by [16]

$$
D_{\perp}=\frac{\left\langle\left\langle\gamma_{\Sigma}\right\rangle\right\rangle}{\left\langle\left\langle k_{y}\right\rangle\right\rangle} \alpha_{\mathrm{D}}(r)
$$

Here, $\gamma_{\Sigma}$ is the sum of growth rates of the excited instabilities and $\langle\langle\cdots\rangle\rangle$ denotes the average value over the $k$-region with positive growth rate. The empirical profile $\alpha_{\mathrm{D}}(r)$ is chosen to be proportional $q^{2}(r)$ [16]. Analogously to the heat diffusivity $D_{\mathrm{h}}$ which is defined by $\chi=n D_{\mathrm{h}}$, where $\chi$ is the heat conductivity, we define a viscosity diffusivity $D_{\eta}$ by $\eta_{2}=\rho D_{\eta}$ where $\rho=n m_{j}$ is the mass density. (The dimension of both diffusivities is $\mathrm{m}^{2} \mathrm{~s}^{-1}$.) To account for the anomalous viscosity we replace in equation (9) $\eta_{2}$ by the sum of $\rho D_{\perp}$ and the neoclassical part (10) as it was proposed in [16]:

$$
\eta_{\text {total }}=\rho D_{\perp}+\eta_{2}
$$

Only L-mode discharges are envisaged. The ratio $\eta_{\text {total }} / \eta_{2} \approx 100$ shows the importance of the turbulence in the momentum transport equation (2).

\section{Fourier analysis of the perturbing magnetic field}

The Fourier sine-coefficients of the radial field $B_{r}$ are given by

$$
B_{m n_{\mathrm{s}}}=\frac{1}{2 \pi^{2}} \int_{0}^{2 \pi} \mathrm{d} \theta \int_{0}^{2 \pi} \mathrm{d} \phi B_{r}(\theta, \phi) \sin (m \theta-n \phi)
$$

An analogous formula holds for the cosine-coefficients $B_{m n_{\mathrm{c}}}$. In the following, the geometrical sum

$$
B_{m n}=\sqrt{B_{m n_{\mathrm{s}}}^{2}+B_{m n_{\mathrm{c}}}^{2}}
$$

of both is envisioned because this sum is decisive for the island width and is almost independent from the phase $\phi_{m, n}=$ $\operatorname{arctg}\left(B_{m n_{\mathrm{c}}} / B_{m n_{\mathrm{s}}}\right)$, defined in equation (34). One hundred gridpoints were chosen in the toroidal and in the poloidal directions, respectively.

\section{Solution method}

Equation (15) is a second order equation for $g$, the normalized toroidal velocity if the definition (16) of $G$ is inserted. This equation is replaced by two first order equations. The first equation is obtained by resolving equation (16) with respect to $\partial g / \partial x$.

$$
\frac{\partial g}{\partial x}=G+\frac{0.107 q^{2}}{1+\left(Q^{2} / S^{2}\right)} \frac{\partial \ln T}{\partial x} \frac{B_{\phi}}{B_{\theta}} h
$$

The second equation is that for $\partial G / \partial x$ which is (almost) identical with (15).

In addition, we have equation (22) for the poloidal rotation. For a given temperature, density and normalized toroidal velocity $g$ this equation is solved for $h$ by means of a solver for transcendental equations. We assume a symmetric streaming of the scrape-off plasma into the divertor. This yields as boundary value $h\left(r=r_{\mathrm{s}}\right)=0$. By two reasons we assume that $g\left(r=r_{\mathrm{s}}\right)=0$ :

1. In the case of ALCATOR C-MOD momentum sources are missing.

2. The friction due to neutrals possibly reduces the boundary value $g\left(r=r_{\mathrm{s}}\right)$ to a low value, even if NBI is used as it is done in many devices.

A shooting procedure, adjusting the boundary value $\mathrm{d} g /\left.\mathrm{d} r\right|_{r=r_{\mathrm{s}}}$, is used to ensure that $\mathrm{d} g / \mathrm{d} r=0$ at the plasma centre.

\section{Results}

Since plasma rotation is here the main subject, results concerning the rotation velocity will be presented first.

The source terms (40) and (61) depend quadratically on the perturbing Fourier components. Therefore, results concerning the Fourier analysis of the magnetic fields generated by the coil configurations of TEXTOR and JET are presented in section 9.2 as well.

\subsection{Plasma rotation}

The input data are those of TEXTOR and JET. The TEXTOR (shot \#91269) data are: $r_{\mathrm{in}}=r_{\mathrm{s}}=46 \mathrm{~cm}, R=175 \mathrm{~cm}$, $T_{\mathrm{i}_{\max }}=1500 \mathrm{eV}, T_{\mathrm{e}_{\max }}=1200 \mathrm{eV}, n_{\max }=5.4 \times 10^{13} \mathrm{~cm}^{-3}$, $\eta=1.6, B_{\phi}=2.23 \mathrm{~T}$. NBI is characterized by $P_{\mathrm{MW}}=0.72$, $E_{\mathrm{keV}}=40$ (deuterons). The plasma current is $I_{\mathrm{p}}=350 \mathrm{kA}$ and the maximum toroidal speed $v_{\mathrm{t}_{\max }}=110 \mathrm{~km} \mathrm{~s}^{-1}$.

The choice of the JET data corresponds to shot \#59316: major radius $R=296 \mathrm{~cm}$, minor half axis $a=84 \mathrm{~cm}$, effective radius $r_{\max }=120 \mathrm{~cm}, T_{\mathrm{i}_{\max }}=11 \mathrm{keV}, n_{\max }=$ $3 \times 10^{13} \mathrm{~cm}^{-3}, \eta_{\mathrm{i}}=1.6, B_{\phi}=5.4 \mathrm{~T}, P_{\mathrm{MW}}=13 \mathrm{MW}$. The plasma current is $I_{\mathrm{p}}=2.0 \mathrm{MA}$ and the maximum toroidal speed $v_{\mathrm{p}_{\max }}=210 \mathrm{~km} \mathrm{~s}^{-1}$. Here, the effective radius is defined by equation (8).

Figures 3 and 4 show the temperature profiles of TEXTOR and JET, respectively. The radii of the gridpoints of the abscissa axis are given in centimetres. This applies also for figures $4-8$.

The maxima of the toroidal velocity (figure 5 for TEXTOR and figure 6 for JET) are reproduced with an accuracy of around $10 \%$.

It is assumed that during the braking process the slip frequency is below the critical value. This can be achieved by adjusting the rotating frequency of the helical field. This requires AC-operation, since an oscillating field can 




Figure 3. The maximum ion temperature in TEXTOR (shot \#91289) is $1.5 \mathrm{keV}$ and the profile which is used as input to the rotation calculation, is close to linear.

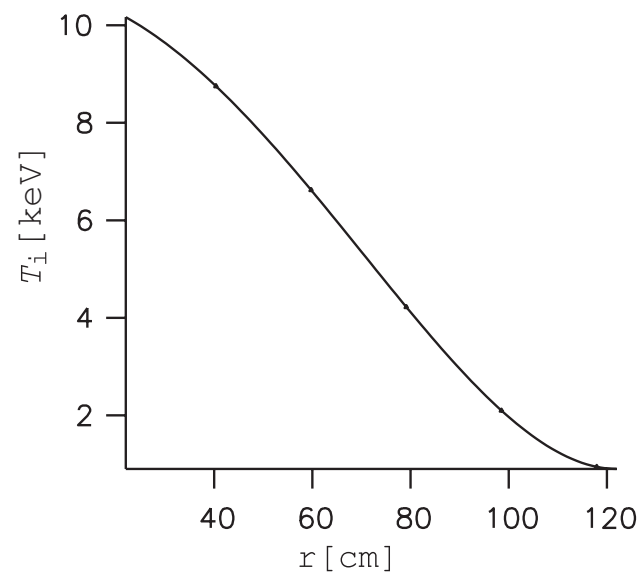

Figure 4. The maximum ion temperature in JET (shot \#59316) is $11 \mathrm{keV}$ and the shape of the profile which is used as input to the rotation calculation as well is similar to that of TEXTOR (figure 3).



Figure 5. The calculated toroidal velocity profile in TEXTOR has the maximum $v_{\phi_{\max }}=120 \mathrm{~km} \mathrm{~s}^{-1}$ (shot \#91289), and the decay is similar to that of the ion temperature in TEXTOR.

be decomposed in two rotating fields with opposite angular velocities. However, AC-operation was not (yet) applied at JET.

At TEXTOR the more efficient operation with four-phase current is possible.

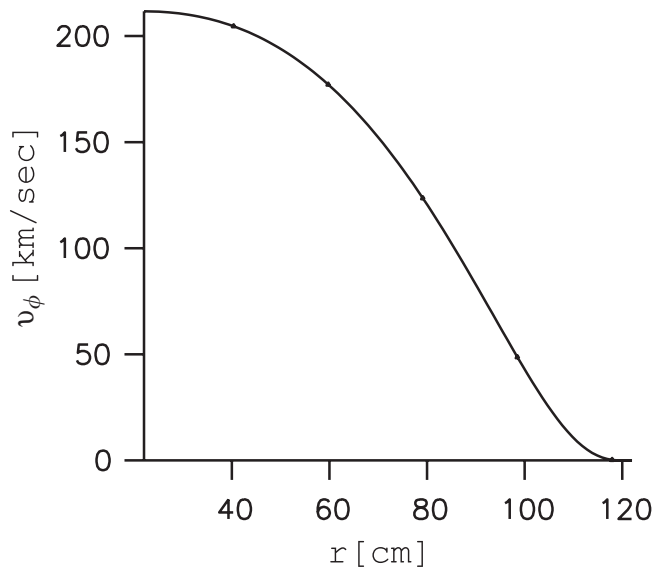

Figure 6. The calculated toroidal velocity profile in JET has the maximum $v_{\phi_{\max }}=220 \mathrm{~km} \mathrm{~s}^{-1}$ (shot \#59316), and the decay is like that of the ion temperature in JET.

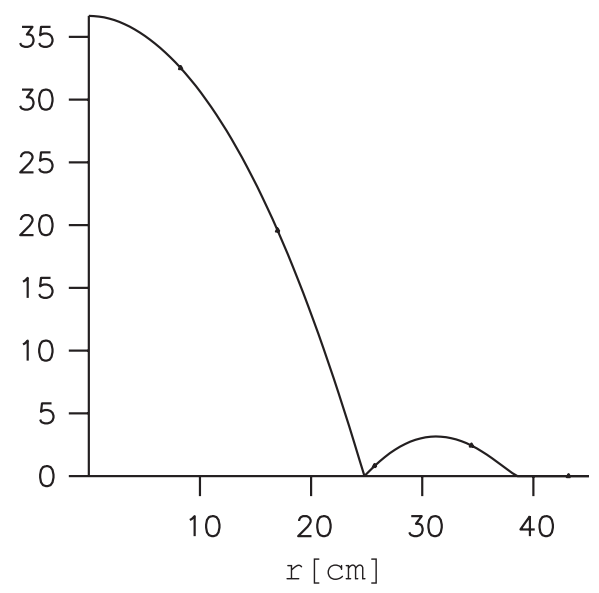

Figure 7. Braking by means of the ( $m=2, n=1)$ Fourier component of the (static) DED leads at TEXTOR to a localized minimum at $r(q=2)=25 \mathrm{~cm}$. In the range $41 \mathrm{~cm}<r<45 \mathrm{~cm}$ the velocity is negative.

At low slip frequencies the source term (61) enforces that the minimum of the (angular) rotation velocity in figures 3-6 is in the vicinity of the rotation velocity of the rotating helical field $\omega_{\mathrm{f}}$. By means of the momentum balance it can be shown that the source term (61) is locally (at $r_{\mathrm{s}}$ ) strongly dominating. Accounting for viscosity and the term (61) the balance yields as amplitude of the (oscillating) radial field

$$
b_{r}=\frac{m R_{0}}{r_{\mathrm{si}} \tau_{\mathrm{si}}} \sqrt{\left(-\Delta^{\prime}\right)^{2} \mu_{0} \tau_{\mathrm{si}} \mu^{\prime}} \frac{1+\left[\left(\omega \tau_{\mathrm{si}}\right)^{2} /\left(-\Delta^{\prime}\right)^{2}\right] \omega_{\mathrm{f}}}{\omega}
$$

where $\mu^{\prime}$ is defined by $1 / \mu^{\prime}=\int_{r_{\mathrm{s}}}^{a}\left(\mathrm{~d} r / r \eta_{2, \text { an }}\right)$. $\eta_{2 \text {, an }}$ is the anomalous viscosity. Using the aforementioned JETdata, assuming $\omega_{\mathrm{f}} \approx 10 \omega$, using $\Delta^{\prime}=-6$ obtained from equation (44) and presupposing the condition (33) one gets the estimate $b_{r} \approx 1.2 \times 10^{-5} \mathrm{~T}$. This is rather close to the 'locking error field' [19]

$$
b_{\mathrm{r}, 1}=\frac{R_{0} \omega_{0}}{2 n r_{\mathrm{si}}} \sqrt{0.5 \mu_{0} \tau_{\mathrm{si}} \mu^{\prime}} \approx 2.7 \times 10^{-5} \mathrm{~T}
$$

by means of which the 'fully reconnected' plasma state at low rotation speed may be obtained. Since the helical field is of the 


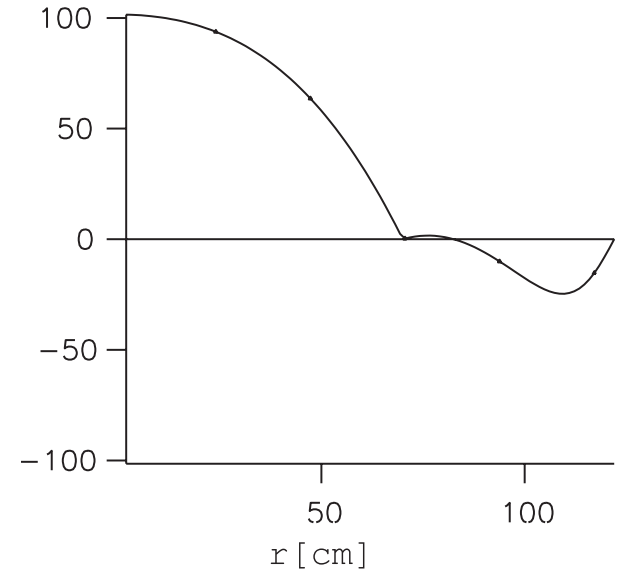

Figure 8. Braking by means of the $(m=2, n=1)$ Fourier component of the saddle coils at JET, the frequency of which is gradually lowered to 0 , leads to a localized minimum at $r(q=2)=70 \mathrm{~cm}$. The stepwise reduction of the frequency is achieved (numerically) by starting step $n$ by the boundary condition of step $(n-1)$. Around ten steps are needed. In

$80 \mathrm{~cm}<r<120 \mathrm{~cm}$ the velocity is negative. Because of the large slip frequency the mode locking could not be seen experimentally.

order of $10^{-3} \mathrm{~T}$, i.e. much larger than $b_{\mathrm{r}, 1}$, the source term $(61)$ is dominant if the slip frequency is small enough.

By gradually reducing the rotation speed of the helical field $\Omega_{\mathrm{f}}$, i.e. by keeping the slip frequency below the limit (33) mentioned in section 4 . the minima can be pulled to the abscissa (figure 7 for TEXTOR and figure 8 for JET) i.e. the (final) rotation speeds of the helical field in figures 7 and 8 are zero at the singular $(q=2)$ surfaces. In figure 8 for $80 \mathrm{~cm}<$ $r<120 \mathrm{~cm}$ the velocity is negative because of the second term on the RHS if equation (16). The analogous dependence in figure 7 has rather small radial extension $(37 \mathrm{~cm}<r<46 \mathrm{~cm})$ and is therefore not shown.

We note that the plasma braking shown in figures 7 and 8 entails a strong reduction of the central velocity, to $\approx \frac{1}{3}$ in the case of TEXTOR and $\approx \frac{1}{2}$ in the case of JET. Since the minima are located at $\approx \frac{1}{2}$ of the effective plasma radius, the mean velocity gradients are roughly the same as in figures 5 and 6 , i.e. the plasma braking does not change the velocity gradient much.

The stepwise reduction of the frequency is achieved (numerically) by starting step $n$ by the boundary condition of step $(n-1)$. Around ten steps are needed.

Because of the large slip frequency the mode locking could not be seen experimentally at JET.

We note that with the same input parameters smooth solutions like in figures 3 and 4 can be obtained because at large slip frequencies the source term is small and does not influence the $v_{\phi}$ profile. The slip frequency turns out to be large if the 'shooting' is started with a boundary value $\left.(\mathrm{d} g / \mathrm{d} r)\right|_{r=r_{\mathrm{s}}}$ close to those of figures 3 or 4 .

The gradient of the toroidal velocity which is approximately equal to the 'shearing rate' defined in [29], is around $4 \times 10^{5} \mathrm{~s}^{-1}(\mathrm{JET})$. For the suppression of the ITG-instability approximately a shearing rate

$$
\omega_{E \times B}>\gamma_{\mathrm{ITG}}
$$

is needed. Using the expression for $\omega_{E \times B}$ in [29] one obtains

$$
\omega_{E \times B}=\frac{B_{\theta}}{B} \frac{\mathrm{d}}{\mathrm{d} r} \frac{E_{r}}{B_{\theta}}
$$

Here, it is assumed that the radial and poloidal correlation lengths of the respective instability (both perpendicular to the magnetic field) are equal.

Due to the large toroidal momentum sources, $v_{\phi} B_{\theta}$ is assumed to be dominating in the pressure balance

$$
E_{r}=v_{\theta} B_{\phi}+v_{\phi} B_{\theta} \approx v_{\phi} B_{\theta}
$$

and thus we obtain

$$
\omega_{E \times B}=\frac{B_{\theta}}{B} \frac{\mathrm{d}}{\mathrm{d} r} v_{\phi}
$$

Therefore, the condition for suppressing the ITG-instability is

$$
\frac{B_{\theta}}{B} \frac{\mathrm{d} v_{\phi}}{\mathrm{d} r}>\gamma_{\mathrm{ITG}}
$$

Since according to [16]

$$
\gamma_{\mathrm{ITG}} \approx 10^{5} \mathrm{~s}^{-1}
$$

the velocity gradient

$$
\frac{\mathrm{d} v_{\phi}}{\mathrm{d} r} \approx 10^{6} \mathrm{~s}^{-1}
$$

is required. Therefore, the velocity gradient achieved is still too small.

\subsection{Magnetic field structure}

The coil configurations of JET and TEXTOR are quite different: at JET (figure 12) the saddle coils located in the lower half of the vacuum vessel are used to produce a broad spectrum of Fourier components. The TEXTOR coils (figure 13) consist of helical conductors at the high field side of the torus [22]. The winding is suited for four phase operation and has the dominant mode at $m=12$ and $n=4$ since it can roughly be understood as cut from complete (12/4)-winding, so that the extension at the high field side is approximately $120^{\circ}$.

9.2.1. JET. By means of the winding generator in FLOC [23] four loops are generated (figures $9(a)$ and $(b)$ ) which represent the saddle coils at JET. As compared with the real configuration (figure 12) some simplifications were done: the curved poloidal connections were approximated by three straight conductors. The finite size of the coil sections in poloidal and toroidal direction (each composed of three filaments) was neglected. Since the lower Fourier components are rather insensitive with respect to geometrical details, the simplifications should be tolerable.

The Fourier components are computed at the $q=2$ surface. For the adjacent $q=3$ and $q=4$ surfaces, the components must, in principle, be recalculated.

The Fourier analysis of the magnetic field of the saddle coils concerns two configurations:

- Antiparallel currents in each of the four modules (figure 9(a)); this leads to dominant Fourier components with $n=2$ because the perturbing poloidal field 



Figure 9. The saddle coils at JET (a complete drawing is shown in figure 12) are replaced by four toroidal and four poloidal conductors. The finite coil thickness was neglected and the conductors are replaced by nine straight filaments in toroidal direction and by three in poloidal direction. Two current configurations have been envisaged: in $(a)$ the currents are antiparallel in adjacent windings, which favours the $n=2$ Fourier components. In $(b)$ the currents in two adjacent coil pairs are parallel, but opposite in the two pairs. This favours $n=1$ Fourier components. The currents in all conductors are $10 \mathrm{kA}$.
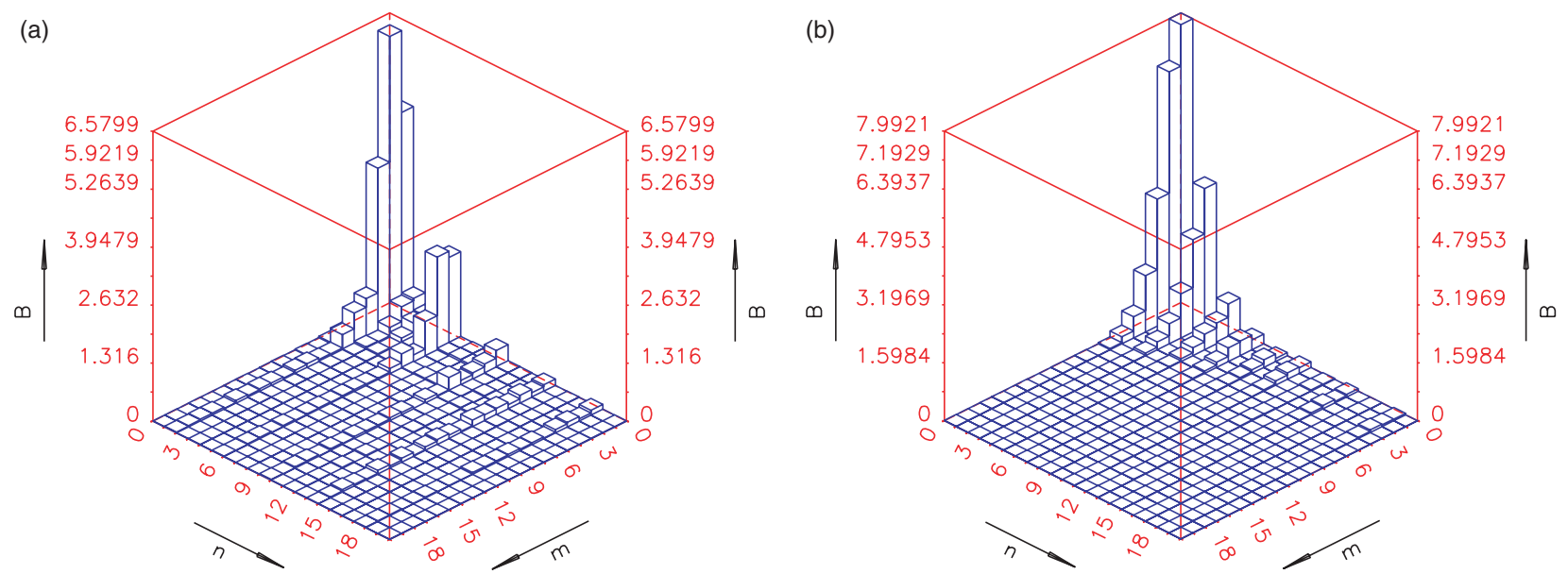

Figure 10. $(a)$ shows the spectrum of the configuration in figure $9(a)$. Here and in the analogous figures, the $(m, n)$ Fourier component is surrounded by the grid lines belonging to the gridpoints $m, m-1, n$ and $n-1$. The dominant component is $B_{\theta_{m=2 n=2}} \approx 6.5 \mathrm{G}$. The analogous component in $(b)$ (belonging to the configuration in figure $9(b))$ is $b_{\theta_{m=2, n=1}} \approx 6.8 \mathrm{G}$. In both cases, the total coil current is $10 \mathrm{kA}$.

component changes four times the sign when going the long way around the torus.

- Parallel currents in adjacent pairs of the four modules (figure $9(b)$ ); the currents in the pairs are opposite. The dominant Fourier components are those with $n=1$ by analogous reasons as before.

The coil current in both configurations is $10 \mathrm{kA}$. Figure 10(a) shows the spectrum of the first configuration. The dominant component is $B_{\theta_{m=2, n=2}} \approx 6.5 \mathrm{G}$.

The spectrum of the second configuration is shown in figure $10(b)$. The component which is resonant at the $q=2$ surface is $B_{\theta_{m=2, n=1}} \approx 7.2 \mathrm{G}$. $B_{\theta_{m=1, n=1}} \approx 9 \mathrm{G}$ is somewhat larger but not resonant at $q=2$. In both cases the total coil current is $10 \mathrm{kA}$. Since resonance at $q=2$ is to be achieved, the second configuration with a large $n=1, m=2$ component is used for the experiments at JET.

According to [18] the braking term must be increased by a factor $646 / q^{2}$ to account for the ' $1 / v$-regime'. Equivalent to that is an increase of the Fourier components by roughly a factor $25.4 / q \approx 10$. We therefore have an effective relative perturbation of $B_{m=2, n=1} / B_{\mathrm{t}}=3 \times 10^{-3}$. We note that the spectra mentioned are rested upon a pure vacuum calculation, i.e. the iron core and the vacuum vessel (possibly important in the case of AC-operation) are neglected.

9.2.2. TEXTOR. The perturbation coils at TEXTOR (figure 13) are foreseen to have the dominant Fourier components in resonance with the $q=3$ surface, i.e. the pitch 

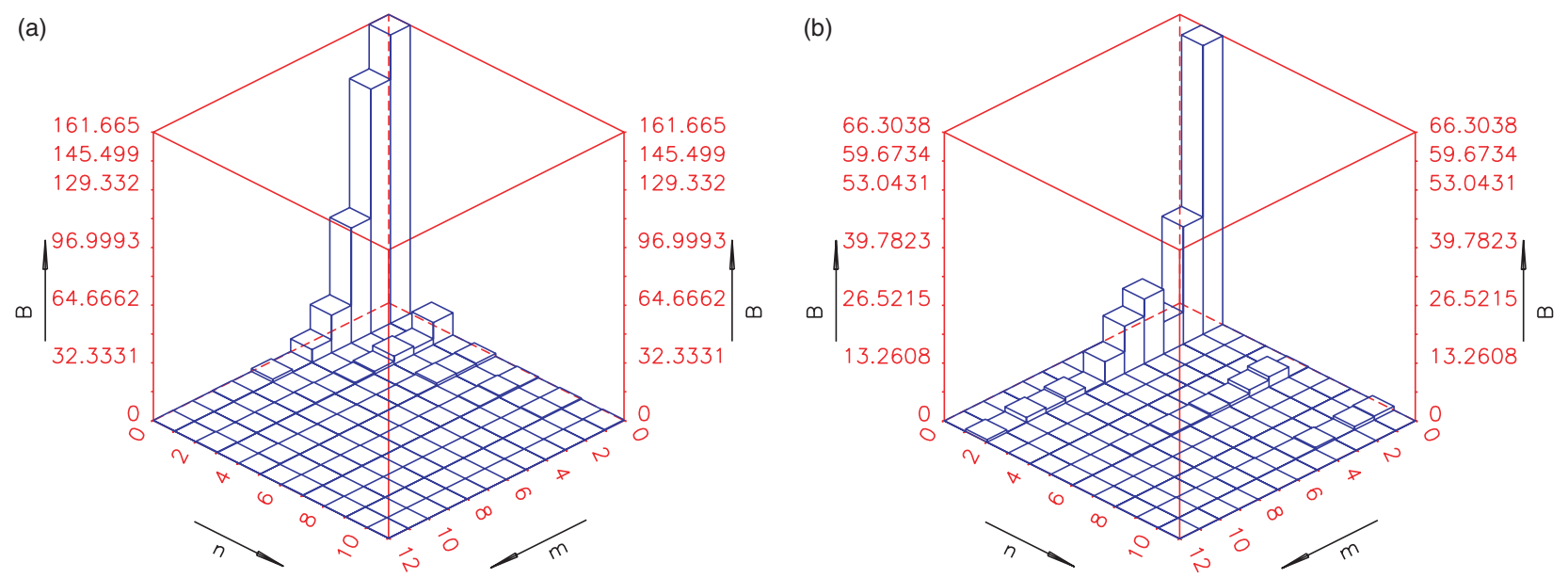

Figure 11. The spectra of the DED at the $q=2$ surface for $I_{\mathrm{d}}=10 \mathrm{kA}$ and $p_{3}=1(a)$ and $p_{2}=1(b)$ are displayed. For $p_{3}=1$ we have an $(m=3, n=1)$ winding and for $p_{2}=1$, an $(m=6, n=2)$ winding. Due to the large distance between the winding and the $q=2$ surface the $(m=1, n=1)$ component and the $(m=2, n=1)$ components are larger than the $(m=3, n=1)$ component. The $(n=2)$ components in $(b)$ show an analogous behaviour.

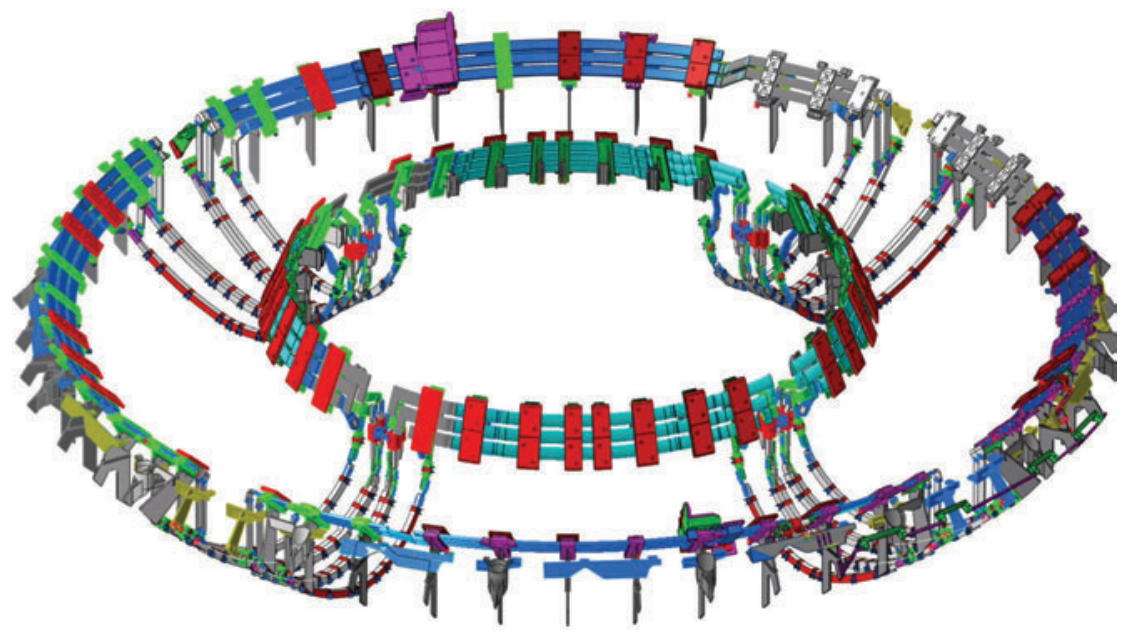

Figure 12. The drawing of the lower saddle coils which are considered here shows the technical details of the two coil pairs schematically shown in figures $9(a)$ and $(b)$. The toroidal and poloidal sections are composed by three conductors (blue, grey or dark green). The support structures are drawn in red or light green.

of the conductors corresponds to the (mean) field line pitch at the $q=3$ surface. To control the penetration depth, the winding can be switched as mixture of a $(m=12, n=4)$, ( $m=6, n=2)$ and a $(m=3, n=1)$ winding. The coil currents are then given by

$$
\begin{aligned}
I_{j}= & I_{\mathrm{d}}\left[\left(1-p_{2}-p_{3}\right) \sin \left(j \frac{2 \pi}{4}+\omega t\right)+p_{2} \sin \left(j \frac{2 \pi}{8}\right)\right. \\
& \left.+p_{3} \sin \left(j \frac{2 \pi}{16}+\omega t\right)\right]
\end{aligned}
$$

$j=1, \ldots, 16$. The mixing factors are $p_{2}=p_{3}=0$ for the ( $m=12, n=4), p_{2}=1, p_{3}=0$ for the $(m=6, n=2)$ and $p_{2}=0, p_{3}=1$ for the $(m=3, n=1)$ winding. Since we are interested in the braking at the $q=2$ surface, the choice $p_{2}=0, p_{3}=1$ is promising because in this case the perturbation at the $q=2$ surface can be expected to be sufficiently large. Figure 11(a) shows the spectrum at the $q=2$ surface for $I_{\mathrm{d}}=10 \mathrm{kA}$. Due to the large distance between the winding and the $q=2$ surface the $m=1, n=1$ component and the $m=2, n=1$ components are larger than the $m=3, n=1$ component. The relative strength of the $m=2, n=1$ component is $b_{m=2, n=1} / 2.23 \mathrm{~T} \approx 0.7 \times 10^{-2}$. Because of the correction due to [18] the effective relative perturbation is around $7 \times 10^{-2}$. Figure $11(b)$ shows the spectrum at the $q=2$ surface for $p_{2}=1, p_{3}=0$ and $I_{\mathrm{d}}=10 \mathrm{kA}$. Now $n=2$ components are generated among which, due to the large distance between the winding and the $q=2$ surface, the $m=1, n=2$ component and the $m=2, n=2$ components are much larger than the $m=6$, $n=2$ component.

\section{Conclusions and discussion}

The inclusion of the braking term due to pressure anisotropization of [17] and the source term due to the $\vec{j} \times \vec{B}$ force density at the singular surface of [19] into the (revisited) neoclassical theory shows that at the singular surface the latter term dominates if the slip frequency is below the critical value 


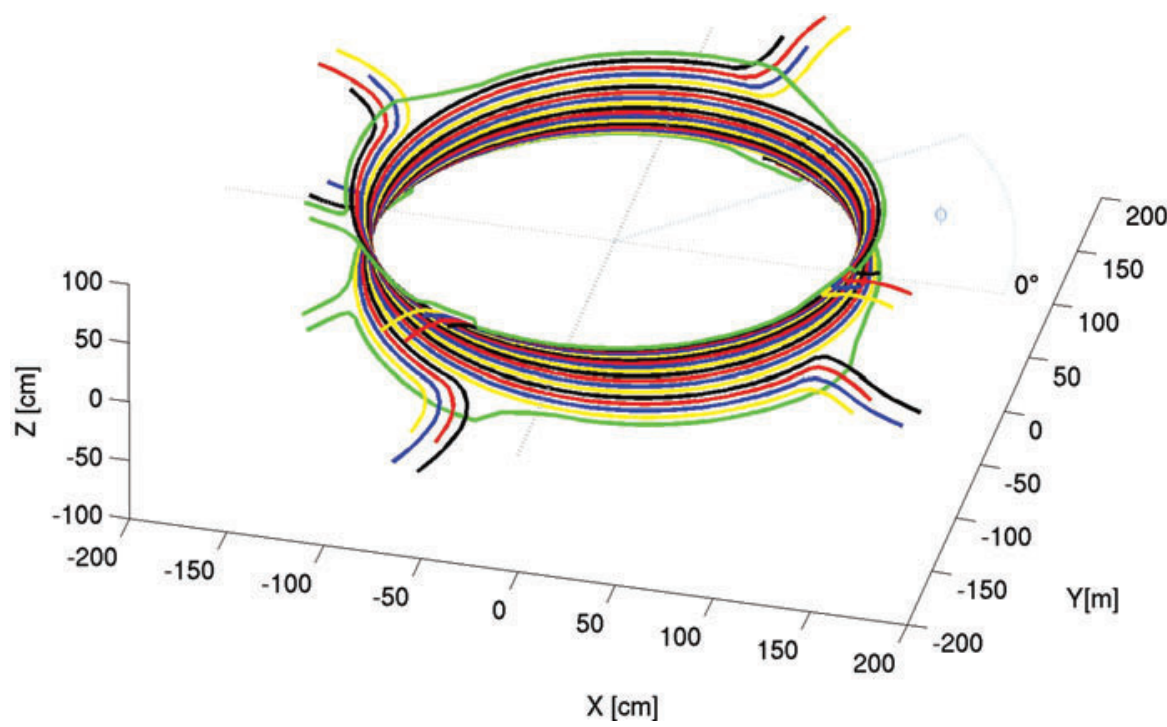

Figure 13. The drawing of the DED coils shows that the winding consisting of 16 conductors with independent feeders can generate the modes $(m=12, n=4),(m=6, n=2),(m=3, n=1)$. In the case of the $(m=3, n=1)$ mode four neighbouring windings are switched in parallel to approximate the current distribution for $p_{3}=1, p_{2}=0$. For the $(6,2)$ mode, two neighbouring conductors are switched in parallel. In the case of the $(12,4)$ winding, each quadruplet (the conductors of which are drawn in black, red, blue and yellow) is fed by four-phase current. The 'compensation' coils (in green) remove the influence of the winding on the radial plasma position.

whereas outside this surface NBI and viscosity losses are important.

In the case of low slip frequency, pressure anisotropization has its main contribution at the singular surface as well, but in the vicinity of this surface the contribution can be considerable corresponding to the $1 /(m-n q(r))$ dependence. The calculations show that there can be a strong impact of weak helical perturbations on the rotation velocity if the slip frequency is below its critical value $\omega_{\text {crit }}=-\Delta / \tau_{\mathrm{si}}$. If this condition is not fulfilled, the rotating fields must be considerably larger than the field required at $\omega=0$ to brake the rotation.

The Fourier analysis shows that in the case of the saddle coils at JET $n=1$ modes appear. Thus, the $(m=2, n=1)$ mode could, in principle, be used to influence the rotation velocity at the $q=2$ surface. However, the large slip frequency due to the DC-operation at JET prevented this. Besides synchronization of the helical field larger currents in the coils could lock the plasma. This, however, is not possible because of the technical limits.

In spite of the locally very strong braking term, it seems to be difficult to produce a strong gradient in the rotation field leading to an ITB, mainly because besides steepening the radial profile also a reduction of the maximum velocity at the plasma centre occurs so that the gain in the velocity gradient is rather small.

In TEXTOR, the perturbation coils allow for large Fourier components with the mode numbers $(m=2, n=1)$ and ( $m=3, n=1)$. Therefore locking was achieved in the DC case by increasing the DED current. Synchronization of the helical fields with the plasma rotation was not needed.

\section{Appendix A. Treatment of the singularity}

The singular denominator in the source term (40) needs a careful treatment in the numerical approach. First, we replace the singularity in $q$ by a singularity in the radius $r$.

$$
\frac{1}{m-n q}=\frac{1}{n(m / n-q)} \approx \frac{1}{n(\mathrm{~d} q / \mathrm{d} r)\left(r-r_{\mathrm{si}}\right)}
$$

Here, $r_{\mathrm{si}}$ is the radius of the surface $q=m / n$. By physical reasons (discussed below) the singularity must be replaced by

$$
f_{\text {sing }}(r)=\frac{1}{\left|r-r_{\mathrm{si}}\right|}
$$

for $\left|r_{\mathrm{si}}-r\right|>\epsilon_{0}$ and

$$
f_{\text {sing }_{1}}(r)=\frac{1}{\epsilon_{0}}
$$

for $\left|r_{\mathrm{si}}-r\right|<\epsilon_{0}$. We approximate this radial dependence by a function typical for resonances

$$
f_{\text {sing }_{2}}(r)=A \frac{1}{\sqrt{\left(r-r_{\mathrm{si}}\right)^{2}+\tilde{\epsilon}^{2}}}
$$

where $\tilde{\epsilon}$ is a small length allowing a numerical treatment. The amplitude $A$ is obtained from the condition that the radial integrals over both source functions should be the same:

$$
\int_{r_{\mathrm{si}}-\epsilon_{1}}^{r_{\mathrm{si}}+\epsilon_{2}} f_{\text {sing }_{1}}(r) \mathrm{d} r=\int_{r_{\mathrm{si}}-\epsilon_{1}}^{r_{\mathrm{si}}+\epsilon_{2}} f_{\text {sing }_{2}}(r) \mathrm{d} r
$$

The distances $\epsilon_{1,2}$ from the singular surface are chosen such that the function $K \times\left(r-r_{\mathrm{si}}\right)$ can be assumed constant. We get, assuming $x=r-r_{\mathrm{si}}$

$$
\begin{aligned}
I_{1} & =\int_{-\epsilon_{1}}^{\epsilon_{2}} f_{\text {sing }} \mathrm{d} r=\int_{-\epsilon_{1}}^{-\epsilon_{0}} \frac{1}{|x|} \mathrm{d} x+\left.\frac{x}{\epsilon_{0}}\right|_{-\epsilon_{0}} ^{\epsilon_{0}}+\int_{\epsilon_{0}}^{\epsilon_{2}} \frac{1}{x} \mathrm{~d} x \\
& =\ln \left(\epsilon_{1}\right)-\ln \left(\epsilon_{0}\right)+2+\ln \left(\epsilon_{2}\right)-\ln \left(\epsilon_{0}\right)=\ln \left(\frac{\epsilon_{1} \epsilon_{2}}{\epsilon_{0}^{2}}\right)+2
\end{aligned}
$$


As integral over the 'resonant' function (72) we obtain

$$
\begin{aligned}
I_{2} & =\int_{r_{\mathrm{si}}-\epsilon_{1}}^{r_{\mathrm{si}}+\epsilon_{2}} f_{\text {sing }_{2}}(r) \mathrm{d} r=A \int_{-\epsilon_{1}}^{\epsilon_{2}} \frac{\mathrm{d} x}{\sqrt{x^{2}+\tilde{\epsilon}^{2}}} \\
= & A \ln \frac{\epsilon_{2}+\sqrt{\epsilon_{2}^{2}+\tilde{\epsilon}^{2}}}{-\epsilon_{1}+\sqrt{\epsilon_{1}^{2}+\tilde{\epsilon}^{2}}}=A \ln \frac{\epsilon_{2}+\epsilon_{2} \sqrt{1+\left(\tilde{\epsilon}^{2} / \epsilon_{2}^{2}\right)}}{-\epsilon_{1}+\epsilon_{1} \sqrt{1+\left(\tilde{\epsilon}^{2} / \epsilon_{1}^{2}\right)}}
\end{aligned}
$$

Expansion with respect to $\tilde{\epsilon}$ yields

$$
\begin{aligned}
I_{2} & =A \ln \frac{\epsilon_{2}+\epsilon_{2}\left(1+0.5\left(\tilde{\epsilon}^{2} / \epsilon_{2}^{2}\right)\right)}{0.5\left(\tilde{\epsilon}^{2} / \epsilon_{1}\right)} \\
& =A \ln \frac{4 \epsilon_{2}+\tilde{\epsilon}^{2} / \epsilon_{2}}{\tilde{\epsilon}^{2} / \epsilon_{1}} \approx A \ln \frac{4 \epsilon_{2} \epsilon_{1}}{\tilde{\epsilon}^{2}}
\end{aligned}
$$

Thus, we get

$$
\int_{-\epsilon_{1}}^{\epsilon_{2}} f_{\text {sing }_{2}} \mathrm{~d} r=A \ln \frac{4 \epsilon_{2} \epsilon_{1}}{\tilde{\epsilon}^{2}}=\int_{-\epsilon_{1}}^{\epsilon_{2}} f_{\text {sing }_{1}} \mathrm{~d} r=\ln \frac{\epsilon_{1} \epsilon_{2}}{\epsilon_{0}^{2}}+2
$$

We assume $\epsilon_{1}=\epsilon_{2}$ and obtain

$$
A \ln \frac{2 \epsilon_{1}}{\tilde{\epsilon}}=\ln \frac{\epsilon_{1}}{\epsilon_{0}}+1
$$

Thus, we have

$$
A=\frac{\ln \left(e \epsilon_{1} / \epsilon_{0}\right)}{\ln \left(2 \epsilon_{1} / \tilde{\epsilon}\right)}
$$

where $e$ is Euler's number. Assuming in addition $\epsilon_{0}=\tilde{\epsilon}$ allows to estimate $A$ as

$$
A=1+\frac{\epsilon_{0}}{\epsilon_{1}}\left[\frac{1}{e}-\frac{1}{2}\right] \approx 1
$$

An estimate of $\epsilon$ can be inferred from the condition that the plasma has to be in the plateau regime.

$$
1 \gg \frac{v_{\mathrm{i}}}{\omega_{T, \mathrm{i}}} \gg\left[\frac{\delta B_{\mathrm{t}}}{B}\right]^{3 / 2},\left[\frac{\delta B_{\mathrm{h}}}{B}\right]^{3 / 2}
$$

$\nu_{\mathrm{i}}$ is the collision frequency and

$$
\omega_{T, \mathrm{i}}=\frac{v_{T_{\mathrm{i}}}}{L_{\mathrm{hel}} / 2 \pi}
$$

the transit frequency, $\delta B_{\mathrm{h}}$ the field variation along the field lines due to the helical perturbation, $\delta B_{\mathrm{t}}$ the field variation along the field lines due to the toroidal variation and $L_{\text {hel }}$ the field line length between two neighbouring magnetic wells due to the helical field, which modulates the tokamak field [27]. The aforementioned condition can be rewritten as

$$
\lambda_{\mathrm{mfp}} \gg L_{\mathrm{hel}}
$$

where $\lambda_{\mathrm{mfp}}=v_{T_{\mathrm{i}}} / \nu_{\mathrm{i}}$ is the mean free path length. Thus a nonresonant surface is still in the plateau regime, if the path length $L_{\text {hel }}$ is smaller than the mean free path length. Since $L_{\text {hel }}$ becomes infinite at the resonant surface, the particles at this surface are in the collision dominated regime. For $T_{\mathrm{i}}=5 \mathrm{keV}$ and $n_{\mathrm{i}}=6 \times 10^{13} \mathrm{~cm}^{-3}$ we get the collision frequency $v_{\mathrm{i}}=100 \mathrm{~s}^{-1}$, the thermal velocity $v_{T_{\mathrm{i}}}=10^{6} \mathrm{~m} \mathrm{~s}^{-1}$ and the mean free path length $=10^{4} \mathrm{~m}$. This is the upper limit of $L_{\text {hel }}$. The distance in $\iota$-space from the singular surface in which the plasma is in the Pfirsch-Schlüter regime, is given by the angle $\Delta \iota$ by which a field line in the singular layer must be turned to obtain this length between the magnetic wells. It is given by

$$
\Delta \iota \lambda_{\mathrm{mfp}}=\frac{2 \pi r_{\mathrm{si}}}{m}
$$

where $2 \pi r_{\mathrm{si}} / m$ is the distance between two adjacent magnetic wells. Thus, we have

$$
\Delta \iota=\frac{2 \pi r_{\mathrm{si}}}{m \lambda_{\mathrm{mfp}}}
$$

and since

$$
\Delta q=-\frac{2 \pi}{\iota^{2}} \Delta \iota=\frac{\mathrm{d} q}{\mathrm{~d} r}\left(r-r_{\mathrm{si}}\right)=1.6 \times 10^{-4}
$$

we get as the distance from the singular surface where the plasma is in the Pfirsch-Schlüter regime

$$
\left|r-r_{\mathrm{si}}\right|<\epsilon_{0}=\frac{1.6 \times 10^{-4}}{\mathrm{~d} q / \mathrm{d} r} \approx 10^{-5} \mathrm{~m}
$$

i.e. smaller than the Larmor radius. Therefore, the fraction of the particles with the guiding centre moving on the singular flux surface must be very small.

\section{Appendix B. Braking time due to ( $m=0, n=1$ )-perturbations driven by mode coupling [17]}

From the momentum balance (without source) we can estimate the time needed to brake down the plasma from a given initial speed. From

$$
m_{\mathrm{i}} n_{\mathrm{i}} \frac{\partial v_{\phi}}{\partial t}+\left\langle\vec{e}_{\phi} \cdot \nabla \Pi\right\rangle=0
$$

we get, using that $\left\langle\vec{e}_{\phi} \cdot \nabla \Pi\right\rangle$ is proportional to $v_{\phi}$,

$$
\frac{\partial v_{\phi}}{\partial t}+\left[\frac{\left\langle\vec{e}_{\phi} \cdot \nabla \Pi\right\rangle}{v_{\phi}}\right] \frac{v_{\phi}}{m_{\mathrm{i}} n_{\mathrm{i}}}=0
$$

The breaking time then reads

$$
\tau=\frac{v_{\phi}}{\left\langle\vec{e}_{\phi} \cdot \nabla \Pi\right\rangle} m_{\mathrm{i}} n_{\mathrm{i}}
$$

For the $m=0$ contribution (which is assumed here to be dominant) we get [17]

$$
K=\frac{4 \sqrt{\pi} p_{\mathrm{i}}}{v_{T_{\mathrm{i}}}} v_{\phi}\left(\frac{\psi_{0, n}}{a B_{\phi}}\right)^{2} \frac{1}{n \epsilon^{2}}
$$

Now we assume (as a reasonable example)

$$
\frac{\psi_{0, n}}{a B_{\phi}}=10^{-3}
$$

and obtain

$$
K=\frac{4 \sqrt{\pi} p_{\mathrm{i}}}{R_{0} v_{T_{\mathrm{i}}}} v_{\phi} 10^{-6} \frac{1}{n \epsilon^{2}}
$$

Therefore,

$$
\tau=\frac{m_{\mathrm{i}} n_{\mathrm{i}}}{K^{*}}
$$

$\left(K^{*}=K / v_{\phi}\right)$ or

$$
\tau=\frac{R_{0} v_{T_{\mathrm{i}}}}{4 \sqrt{\pi} p_{\mathrm{i}}} 10^{6} n \epsilon^{2} m_{\mathrm{i}} n_{\mathrm{i}}
$$


With the scalar pressure

$$
p_{\mathrm{i}}=n_{\mathrm{i}} k T_{\mathrm{i}}
$$

and the thermal velocity

$$
v_{T_{\mathrm{i}}}=\sqrt{\frac{k T_{\mathrm{i}}}{m_{\mathrm{i}}}}=0.99 \times 10^{4} \sqrt{\frac{T_{\mathrm{i}}}{M}}\left[\mathrm{~m} \mathrm{~s}^{-1}\right]
$$

(where $M$ is the ion mass in amu) we can write

$$
\tau=\frac{R_{0} v_{T_{\mathrm{i}}}}{4 \sqrt{\pi} v_{T_{\mathrm{i}}}^{2}} 10^{6} n \epsilon^{2}
$$

or

$$
\tau=\frac{R_{0}[\mathrm{~m}]}{4 \sqrt{\pi} 0.99 \times 10^{4} \sqrt{T_{\mathrm{i}}[\mathrm{eV}] / M}} 10^{6} n \epsilon^{2}[\mathrm{~s}]
$$

where $R_{0}[\mathrm{~m}]$ is the major radius in metres. With the parameters $\left(R_{0}[\mathrm{~m}]=3, T_{\mathrm{i}}=5 \mathrm{keV}, n=1, \epsilon=0.3\right)$ we have $\tau=67 \mathrm{~ms}$.

\section{Appendix C. Evaluation of integrals over the poloidal coordinate $\chi$}

The poloidal line element $\mathrm{d} l_{\chi}$ is connected to $\mathrm{d} \chi$ by

$$
\mathrm{d} l_{\chi}=h_{\chi} \mathrm{d} \chi
$$

Thus, $h_{\chi}$ depends on the definition of $\chi$.

Since the line element can be obtained easily by following the poloidal circumference of a flux surface in the rectangular grid of a box enclosing the plasma, we choose $\mathrm{d} \chi$ proportional to $\mathrm{d} l_{\chi}$

$$
\mathrm{d} l_{\chi} \sim \mathrm{d} \chi
$$

where $\chi$ is running in $0<\chi<2 \pi$. We get by integrating along the flux contour line

$$
h_{\chi}=\frac{U}{2 \pi}=\mathrm{const}
$$

Thus, we have

$$
\chi=\frac{2 \pi}{U} \int_{0}^{L} \mathrm{~d} l_{\chi}
$$

where $L$ is the length of the contour line. $\chi$, however, is the geometrical angle $\theta=\operatorname{arctg}(y / x)$ only in the case of circular flux surfaces with negligible Shafranov shift. ( $x$ and $y$ are the Cartesian coordinates of the integration point.)

We note that the choice $\mathrm{d} \chi=\mathrm{d} \theta$ leads to

$$
\frac{r_{\text {surf }} \mathrm{d} \theta}{\cos (\alpha)}=\mathrm{d} l_{\chi}
$$

and thus to

$$
h_{\chi}=\frac{r_{\text {surf }}}{\cos (\alpha)}
$$

where $r_{\text {surf }}=\sqrt{x^{2}+y^{2}}$ is the distance between the magnetic axis and the integration point and $\alpha$ the angle between the plasma circumference and the circle around the magnetic axis with radius $r_{\text {surf }}$.

\section{References}

[1] Stringer T.E. 1969 Phys. Rev. Lett. 22770

[2] Rosenbluth M.N. and Taylor J.B. 1969 Phys. Rev. Lett. 23367

[3] Shaing K.C. and Crume E.C. Jr 1989 Phys. Rev. Lett. 63 2369

[4] Craddock G.G. and Diamond P.H. 1991 Phys. Rev. Lett. 67 1535

[5] Burrell K.H. 1997 Phys. Plasmas 41499

[6] Fukuyama A., Takatsuka S., Itoh S.-I., Yagi M. and Itoh K. 1998 Plasma Phys. Control. Nucl. Fusion 40653

[7] Hassam A.B. and Antonsen T.M. Jr 1994 Phys. Plasmas 1337

[8] Coppi B. 2002 Nucl. Fusion 421

[9] Rogister A. 1994 Phys. Plasmas 1619

[10] Claassen H.A., Gerhauser H., Rogister A. and Yarim C. 2000 Phys. Plasmas 73699

[11] Nicolai A., Rogister A.L. and Daybelge U. 2002 Contrib. Plasma Phys. 42241

[12] Rogister A.L., Rice J.E., Nicolai A. and Ince-Cushmann A., Gangadhara S. and Alcator C-Mod group 2002 Nucl. Fusion 421144

[13] Rogister A.L. 1999 Phys. Plasmas 6200

[14] Braginskii S.J. 1966 Transport processes in a plasma Review of Plasma Physics (New York: Consultants Bureau) vol 1, p 205

[15] Mikhailowski A.B. and Tsypin V.S. 1984 Beitr. Plasmaphys. 24335

[16] Tokar M.Z. et al 1999 Plasma Phys. Control. Fusion 41 B317

[17] Lazzaro E. et al 2002 Phys. Plasmas 93906

[18] Lazzaro E. and Zanca P. 2003 Phys. Plasmas 102399

[19] Fitzpatrick R. 1992 Theory of fusion plasmas Proc. Joint Varenna-Lausanne International Workshop (Varenna, 1992) p 147

Fitzpatrick R. 1995 Phys. Plasmas 21648

[20] Fitzpatrick R. 1993 Nucl. Fusion 331049

[21] Santagiustina A. et al 1996 Design of a $m=2, n=1$ tearing mode control system for JET Proc. 19th Symp. on Fusion Technology (Lisbon, Portugal)

[22] Finken K.H. et al 1997 Special issue on: Dynamic ergodic divertor Fusion Eng. Des. 37 341-52

[23] Nicolai A. 1984 J. Nucl. Mater. 121385

[24] Shaing K.C., Hirshman S.P. and Callen J.D. 1986 Phys. Fluids 29521

[25] Jaspers R. 2002 private communication, FZ Jülich

[26] Rice J.E. et al 2000 Phys. Plasmas 71825

[27] Coronado M. and Wobig H. 1988 Phys. Fluids 29527

[28] Audenaerde K., Emmert G.A. and Gordinier M. 1978 Report UWFDM-259, University of Wisconsin

[29] Hahm S.T. and Burrell K.H. 1995 Phys. Plasmas 21648 